\title{
Ordering in ternary nitride semiconducting alloys
}

\author{
Michał Łopuszyński \\ Interdisciplinary Center for Mathematical and Computational Modeling, \\ University of Warsaw, Pawińskiego 5A, 02-106 Warsaw, Poland
}

Jacek A. Majewski

Institute of Theoretical Physics, Faculty of Physics, University of Warsaw, Hoża 69, 00-681 Warsaw, Poland

(Dated: November 16, 2018)

\begin{abstract}
We present a thorough theoretical study of ordering phenomena in nitride ternary alloys $\mathrm{Ga}_{x} \operatorname{In}_{1-x} \mathrm{~N}$, $\mathrm{Al}_{x} \mathrm{In}_{1-x} \mathrm{~N}$, and $\mathrm{Al}_{x} \mathrm{Ga}_{1-x} \mathrm{~N}$. Using the Monte Carlo approach and energetics based on the Keating model we analyze the influence of various factors on ordering in bulk crystals and epitaxial layers. We characterize the degree of both short-range order (SRO) and long ranger order (LRO) for different compositions, temperatures and for substrates associated with different epitaxial strain. For the description of the SRO, the Warren-Cowley parameters related to the first four coordination shells are used. The LRO is detected by means of the introduced sim-LRO parameter, based on the BraggWilliams approach. The description of the observed long-range ordering patterns and conditions for their occurrence follows.

PACS numbers: 61.66.Dk, 61.72.uj, 64.60.De, 64.60.Cn, 81.30.Hd
\end{abstract}

\section{INTRODUCTION}

Ordering in semiconducting nitride alloys is recently an intensively investigated issue. Both experimental as well as theoretical activities deal with mixtures of AlN, GaN, and InN, which are important from the application perspectives such as optoelectronics, high-power and high frequency electronics, and sensors. There are three essential issues that trigger such high research interest.

First, there is much experimental controversy over which ordering patterns can be obtained in nitrides and under what conditions do they occur. Actually, various modes of ordering are reported to be observed in the nitride samples. These include clustering and precipitation, $\frac{1 \mid 2}{\sqrt{2}}$ compositional modulation,,$[4]$ or uniform random alloy possibly with some degree of short-range ordering. 5 However, many of the aspects here still remain unclear. For example, recently Galtrey and coworkers,, on the basis of three dimensional atomic probe (3DAP) measurements, concluded that there is no evidence of clustering in $\mathrm{Ga}_{x} \operatorname{In}_{1-x} \mathrm{~N}$ except for the natural spatial fluctuations of composition. Based on a previous report by Smeeton, 8 they suggested that observed "clusters" could be artifacts related to the radiative damage of the sample during high electron resolution transmission microscopy (HRTEM) observation. However, this argumentation met with serious critique from TEM experimental groups, ${ }^{9] 10}$ pointing out that the radiative damage impact can be highly minimized throughout the experiment and that during such careful measurements clustering of In atoms still remains visible. In the presence of such ambiguities, modeling could provide a valuable insight into the nature of ordering occurring in nitrides.

The second reason is related to the fact that ordering influences the electronic structure and the optical proper- ties of alloys. These properties, including band gap, carrier localization, mobility, etc., are in turn crucial from the applications viewpoint, which include laser diodes (LDs), light-emitting diodes (LEDs), high-electron mobility transistors (HEMTs), sensors, etc. It turns out that even local short-range ordering significantly influences optical properties of nitrides as well as other semiconducting systems, see e.g. the work of Bellaiche et al!11 Of course, global long-range ordering has also a pronounced impact on optical properties in nitride alloys, as it was demonstrated, e.g., in Ref. 12. Recently, also Gorczyca and coworkers published a series of papers comparing electronic structure of small supercells calculated assuming either clustered or uniform distribution of cations 13,15 Their studies show significant differences for these extreme distribution cases, for materials including ternary nitride alloys $\mathrm{Ga}_{x} \operatorname{In}_{1-x} \mathrm{~N}, \mathrm{Al}_{x} \operatorname{In}_{1-x} \mathrm{~N}$, and $\mathrm{Al}_{x} \mathrm{In}_{1-x} \mathrm{~N}$ as well as selected compositions of quaternaries $\mathrm{Al}_{x} \mathrm{Ga}_{y} \mathrm{In}_{1-x-y} \mathrm{~N}$. Yet, another important example of ordering significance for electronic properties is related to the luminescence intensity of In containing nitride samples. There are indications that excitonic recombination occurring at In microclusters could significantly contribute to the luminescence signal.16 17 Therefore, the presence or absence of In clusters should have considerable impact on efficiency of light-emitting devices.

The third issue has methodological origin. The type and degree of homogeneity in the considered alloy influences the range of methods that can be applied to modeling its properties. Many theoretical approaches assume the perfectly random uncorrelated alloy, characterized by both short- and long-range order parameters equal to zero. Examples of such methods include virtual crystal approximation (VCA), coherent potential approximation (CPA), special quasirandom structures approach (SQS), etc. The question about the presence of correlations and 
ordering within nitride alloys is, therefore, also very vital in terms of accuracy and applicability of various modeling schemes. Moreover, the detailed knowledge about the ordering patterns could provide more realistic atomistic configuration for input to semiempirical electronic structure computation methods such as tight-binding or empirical pseudopotential schemes. Thus, the overall reliability of nitride alloys modeling can be seriously improved, once more detailed knowledge about ordering is available.

Keeping in mind the above reasons, we try to shed some light on the ordering phenomena occurring in nitrides. We carry out the detailed studies of ordering in different nitride ternary alloys including $\mathrm{Ga}_{x} \operatorname{In}_{1-x} \mathrm{~N}$, $\mathrm{Al}_{x} \mathrm{In}_{1-x} \mathrm{~N}$, and $\mathrm{Al}_{x} \mathrm{Ga}_{1-x} \mathrm{~N}$. We verify how various factors could influence the ordering in these systems. The crucial variables include composition, temperature, and epitaxial strain. Our simulations are performed under assumption of the thermodynamics equilibrium. Therefore, we do not directly address the effects specific to the growth method. The growth of nitride layers is often performed using methods operating in non-equilibrium conditions such as molecular beam epitaxy (MBE). Moreover, usually this epitaxial growth takes place on the surface. Therefore, the features related to surface ordering and details of growth process could in principle remain "quenched" in the sample, forming a metastable state. Phenomena of this kind are not directly included in our modeling. The main computational tool of our investigation is the static Monte Carlo method. This is not the only option. There are also kinetic studies available in the literature for nitrides $\frac{18}{18}$ The energetics in our calculations is computed on the basis of the Keating valence force field model and, for the sake of simplicity, we focus on the zinc-blende structures. The studies are carried out in the lattice-coherent thermodynamics regime, i.e., we assume that the alloy constituents form common lattice and can not decay into separate components, each with its own independent lattice parameter. This assumption was often not included and there were numerous studies examining the behavior in the so called lattice-incoherent case. These approaches rely mainly on the free-energy difference between alloy and binary constituents relaxed to their own lattice constants. Examples of this type of calculation for ternary $y \underline{19}\left[28\right.$ and for quaternary nitrides ${ }^{29 \mid 30}$ are present in the literature. These studies provided qualitatively similar phase diagrams with unimodal curves separating the uniform region in the composition-temperature parameter space. Moreover, they predicted very high critical temperatures $T_{\mathrm{M}}$, above which the alloy components are fully miscible. For the most analyzed case of the ternary $\mathrm{Ga}_{x} \mathrm{In}_{1-x} \mathrm{~N}$, typical reported values of $T_{M}$ are in the range of $T_{M} \approx 1500-2000 \mathrm{~K}$ (see the work of Liu and Zunger ${ }^{31}$ for a thorough review of different model findings). These high $T_{M}$ values yield very low estimates for the maximum In composition, which can be incorporated in $\mathrm{Ga}_{x} \mathrm{In}_{1-x} \mathrm{~N}$ in typical growth temperatures, be- fore phase separation occurs. On the other hand, there are experimental findings showing that it is possible to grow $\mathrm{Ga}_{x} \mathrm{In}_{1-x} \mathrm{~N}$ samples containing up to $20-30 \%$ of $\mathrm{In}$ without triggering significant clustering, which is in disagreement with aforementioned predictions of the latticeincoherent case. Therefore, recently also a different approach was introduced,,$\sqrt[31]{33}$ where the lattice coherence is assumed. It turned out that in this case $T_{\mathrm{M}}$ decreases to values below typical growth temperatures. Instead of the phase separation, the random alloy phase with some degree of short-range order is predicted. Therefore, in our study, we focus on lattice-coherent case, which seems to correspond better to the alloys obtained during epitaxial growth. Moreover, we also analyze the influence of the stress related to different epitaxial substrates employed, as this can be an important factor suppressing or triggering ordering.22123/28|31/34

In order to characterize the degree of ordering we examine equilibrium structures obtained from our Monte Carlo simulations. To quantify the degree of short-range order in the generated samples we use the Warren-Cowley family of parameters. For description and detection of long-range ordering we employ the approach based on the Bragg-Williams long range order measure. We present a complete and systematic study of these parameters in the whole composition range for all ternary combinations $\mathrm{Ga}_{x} \mathrm{In}_{1-x} \mathrm{~N}, \mathrm{Al}_{x} \mathrm{In}_{1-x} \mathrm{~N}$, and $\mathrm{Al}_{x} \mathrm{Ga}_{1-x} \mathrm{~N}$. We also examine different temperatures, beginning from growth temperature range to higher values which could correspond to the sample annealing conditions. Moreover, we examine how the above ordering metrics are influenced by biaxial strain related to application of a different substrate for growth process.

The paper is organized as follows. We start with a short overview of the short- and long-range ordering concepts in Sec. III. The commonly employed Warren-Cowley and Bragg-Williams parameters are also briefly discussed there. Next, in Sec. III the details of the employed computational approach are explained. Section [V] describes how the order parameters are computed from the Monte Carlo data. In particular, it introduces the simLRO parameter, constructed on the basis of the BraggWilliams characteristic. The sim-LRO metric proposed in this work is a handy indicator of the long-range ordering emergence during Monte Carlo simulations. The results for the ternary bulk alloys $\mathrm{Ga}_{x} \operatorname{In}_{1-x} \mathrm{~N}, \mathrm{Al}_{x} \operatorname{In}_{1-x} \mathrm{~N}$, and $\mathrm{Al}_{x} \mathrm{Ga}_{1-x} \mathrm{~N}$ are described in Sec. $\mathrm{V}$ The ordering in biaxially strained epitaxial $\mathrm{Ga}_{x} \mathrm{In}_{1-x} \mathrm{~N}$ layers is studied in Sec. VI. Finally the paper is concluded in Sec. VII.

\section{QUANTITATIVE DESCRIPTION OF ORDERING}

The concepts of order and disorder are intuitively easily understood. However, there exist many ways of converting this intuition to numbers describing the degree of ordering in crystals. Therefore, to provide a neces- 
sary background and fix the notation, a brief description of the concepts used throughout the forthcoming analysis of ordering in nitrides is given in this section. For more thorough insight into the ordering phenomena, see classical monographs of Ziman,,$[35]$ Ducastelle,,$[36$ or a very informative introductory paper by Klein. ${ }^{37}$

\section{A. Long-range order vs short-range order}

When analyzing the ordering in alloys, it is important to distinguish between two different aspects of this phenomena, namely, the short-range order (SRO) and the long-range order (LRO). The term short-range order is used to describe the preference of certain types of atoms to reside near each other. This effect manifests itself in the form of statistical correlations between occurrences of atomic types on their respective coordination shells. Usually, alloys exhibit non-zero degree of short-range order, as a result of energetic preference towards particular atomic arrangements. The long-range order is related to the development of a global pattern spread throughout the whole crystal. It is worth underlining that in the temperatures above $0 \mathrm{~K}$ the observed pattern is never perfect and contains some misplaced atoms. Both types of ordering can be described in a quantitative manner. In the following we recall the definitions of the commonly used Warren-Cowley SRO parameter and the Bragg-Williams LRO characteristic.

\section{B. Warren-Cowley short-range order parameter}

One of the most commonly used measures of SRO is the Warren-Cowley family of parameters $\Gamma \Gamma^{[38} \mathrm{In}$ multicomponent alloys, these SRO parameters between components $\mathrm{A}$ and $\mathrm{B}$ are defined on the basis of conditional probability of finding an A-type atom in the specified coordination shell of a B-type atom:

$$
\Gamma_{\mathrm{AB}}^{(i)}=1-\frac{P(A \text { atom in shell } i \text { of site } j \mid B \text { atom on site } j)}{c_{\mathrm{A}}}
$$

where $c_{\mathrm{A}}$ denotes the concentration of A-type atoms in the system. It is straightforward to note that the relation $\Gamma_{\mathrm{AB}}^{(i)}<0$ indicates the preference to $\mathrm{AB}$ neighborhood on the $i$ th coordination shell, whereas $\Gamma_{\mathrm{AB}}^{(i)}>0$ indicates anti-preference. For the ideal uncorrelated random alloy, $\Gamma_{\mathrm{AB}}^{(i)}=0$. It can be also shown that ${ }^{36}$

$$
\Gamma_{\mathrm{AB}}^{(i)}=\Gamma_{\mathrm{BA}}^{(i)}
$$

and that for each of the components $\mathrm{P}$ the following sum rule holds:

$$
\sum_{B} c_{\mathrm{B}} \Gamma_{\mathrm{PB}}^{(i)}=0
$$

Therefore, for the alloy $\mathrm{A}_{x} \mathrm{~B}_{1-x}$, we can in principle define four different $\mathrm{SRO}$ measures $\Gamma_{\mathrm{AA}}, \Gamma_{\mathrm{AB}}, \Gamma_{\mathrm{BA}}$, and $\Gamma_{\mathrm{BB}}$. However, because of one symmetry relation [Eq. (2)] and two sum rules [Eq. (3)], actually only one SRO parameter is really independent. In this paper, we use standard convention and focus on $\Gamma_{A B}$. It is worth underlining that, even though technically we deal with ternary alloys $\mathrm{A}_{x} \mathrm{~B}_{1-x} \mathrm{~N}$, they can be described identically as in the binary case. This is because nitrogen lattice remains mostly unaffected, as the probability of substitution in the nitrogen site is much lower than exchanges in cationic lattice because of the energetic reasons. Therefore, we focus only on the cationic fcc sublattice and investigate in a great detail order parameters related to the first four coordination shells within this lattice.

\section{Bragg-Williams long-range order parameter}

The long-range order must be specified with respect to a certain pattern. To calculate it, e.g., for $\mathrm{A}_{x} \mathrm{~B}_{1-x}$ binary alloy we have to know which sites should be occupied by atoms of type A, type B, etc. Once we know the spatial pattern (PAT), we can define the Bragg-Williams longrange order parameter as

$$
S_{\mathrm{A}}^{(\mathrm{PAT})}=\frac{f_{A}^{(\mathrm{PAT})}-c_{\mathrm{A}}}{1-c_{\mathrm{A}}} .
$$

For the SRO parameter, a key role was played by the conditional probability, whereas for the LRO measure the most important variable is $f_{A}^{(\mathrm{PAT})}$. It denotes the fraction of A sites from pattern PAT, which are actually occupied by A type atoms in the structure under consideration. Symbol $c_{\mathrm{A}}$ stands here for concentration of $\mathrm{A}$ atoms in the sample. If $S_{\mathrm{A}}^{(\mathrm{PAT})} \approx 1$, it indicates that the location of A atoms in the structure is similar to pattern PAT. If $S_{\mathrm{A}}^{(\mathrm{PAT})}=0$, it means that no significant similarity to PAT in terms of A-atom locations was detected. From $S_{\mathrm{A}}^{(\mathrm{PAT})}<0$, it can be deduced that $\mathrm{A}$ atoms in the structure avoid A sites from PAT. For the binary alloys $\mathrm{A}_{x} \mathrm{~B}_{1-x}$, the $S_{\mathrm{A}}^{(\mathrm{PAT})}=S_{\mathrm{B}}^{(\mathrm{PAT})}$, so we have only one independent Bragg-Williams LRO parameter. For the technical reasons, the direct use of the Bragg-Williams parameter for the quantification of the LRO in Monte Carlo simulation data is difficult. Therefore, to detect the presence of the LRO in our results, we develop a derived quantity, the sim-LRO parameter. It is described in greater detail in Sec. IV.

\section{COMPUTATIONAL DETAILS}

All calculations, were performed for the zinc blende $6 \times 6 \times 6$ supercell containing 1728 atoms. As resulted from our tests, this size of the supercell ensured good convergence of the examined order parameters. See 


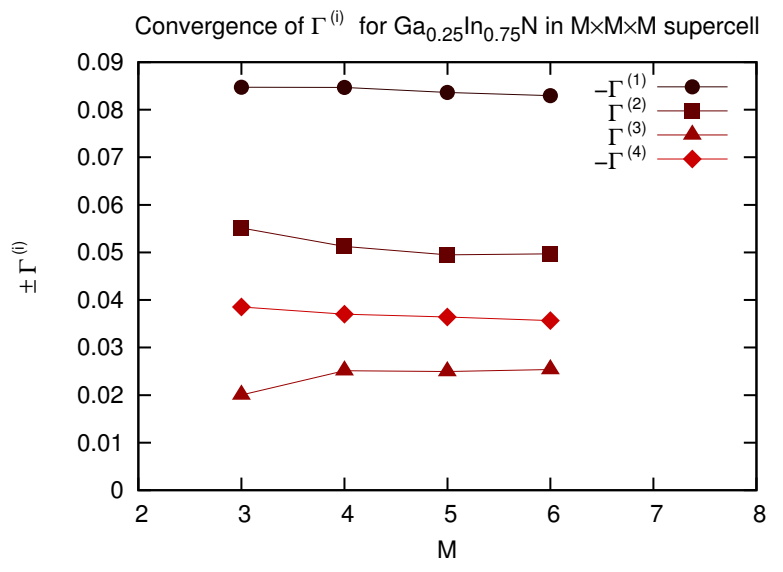

FIG. 1. (Color online) Sample convergence test — the dependence of the Warren-Cowley short-range order parameter $\Gamma_{\text {GaIn }}^{(i)}$ on the $M \times M \times M$ supercell size in bulk $\mathrm{Ga}_{0.25} \operatorname{In}_{0.75} \mathrm{~N}$.

Fig. 1 for a sample test, showing the dependence of $\Gamma_{\text {GaIn }}^{(i)}$ in bulk $\mathrm{Ga}_{0.25} \mathrm{In}_{0.75} \mathrm{~N}$ on the simulation supercell size. When it comes to the applied simulation techniques, the standard static Metropolis algorithm within the NVT ensemble was applied. The concentration of species within alloy was held constant during the simulation, atomic shifts and cationic exchanges were employed as trial moves. Because of the employment of atomic shifts the lattice vibrational effects are directly included in the performed calculations. The magnitude of atomic shifts was adjusted so that exchange probability was equal to 0.5 , as recommended for the most efficient phase space exploration ${ }^{[3940}$ The exchange trials were performed five times more often than atomic shifts, since in the majority of the cases their probability was much lower than for shifts. The total length of simulation was 8.3 million Monte Carlo sweeps per concentration. For each run, we allowed 0.4 million Monte Carlo sweeps for equilibration, before gathering the simulation statistics. The energy calculations were carried out using Keating mode ${ }^{41}$ and its parametrization for nitrides described in the earlier work of the authors. $\underline{42}$ Periodic boundary conditions were used throughout all presented simulations. For every type of ternary alloy, $\mathrm{A}_{x} \mathrm{~B}_{1-x} \mathrm{~N}$ a series of simulations with 17 different concentrations $x=0.056,0.111,0.167, \ldots, 0.944$ was performed. For the initial conditions, random and uncorrelated structures were generated with lattice constant optimized to their energy minimum. For the pseudorandom number generator, the RanLux method was used as implemented in the GSL library $\stackrel{43}{43}$ Also tests with different algorithms were performed, showing that the simulation results are insensitive to pseudorandom number generation technique employed. Figure 2 displays the difference between the $\Gamma_{\text {GaIn }}^{(i)}$ parameters computed for $\mathrm{Ga}_{x} \operatorname{In}_{1-x} \mathrm{~N}$ on InN using the aforementioned RanLux algorithm and Wichmann-Hill method implemented in the ACML library, ${ }^{44}$ This difference was calculated according

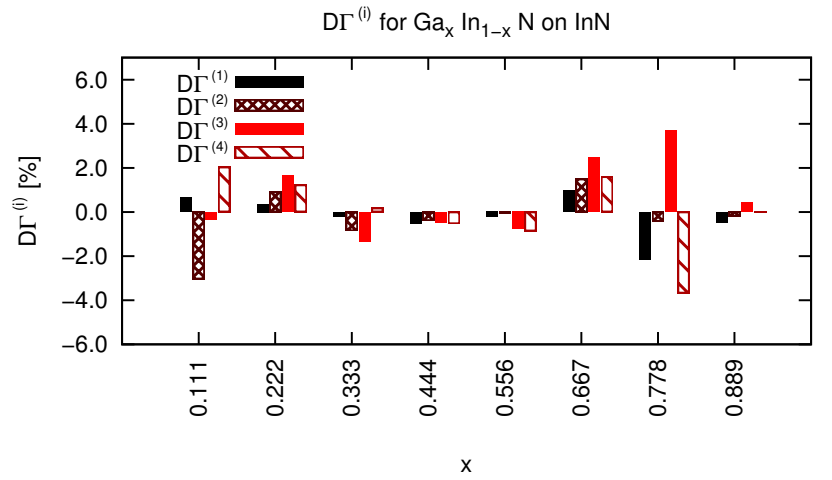

FIG. 2. (Color online) Differences between short-range order parameters estimation from simulations powered by two different random number generator - RanLux from the GSL library and Wichmann-Hill from the ACML package. Simulations were carried out for the $\mathrm{Ga}_{x} \operatorname{In}_{1-x} \mathrm{~N}$ on InN substrate in $T=873 \mathrm{~K}$.

to the formula

$$
D \Gamma_{\text {GaIn }}^{(i)}=\Gamma_{\text {GaIn }}^{(i)}(\text { RanLux })-\Gamma_{\text {GaIn }}^{(i)}(\text { Wichmann-Hill }) .
$$

The observed values of $D \Gamma_{\text {GaIn }}^{(i)}$ are small. As it can be seen from Fig. 2, they do not exceed a few percent.

\section{DETERMINATIONS OF ORDER PARAMETERS FROM SIMULATION DATA}

The computation of Warren-Cowley parameters $\Gamma_{\mathrm{AB}}^{(i)}$ from structures obtained in Monte Carlo simulations is relatively easy. It is enough to estimate the conditional probability from formula (1) by average percentage of type A atoms present in the $i$ th shell of the $\mathrm{B}$ atoms in the set of structures generated during each Monte Carlo run. However, as opposed to the short-range order, the situation for the long-range ordering is not so straightforward. To be able to estimate the Bragg-Williams measure $S_{\mathrm{A}}^{(\mathrm{PAT})}$, the pattern PAT has to be known in advance. Its correct selection is crucial for the detection of the long-range order. When calculating $S_{\mathrm{A}}^{(\mathrm{PAT})}$ for a highly ordered structure, however, very different from assumed PAT, one may easily obtain values close to zero, indicating the absence of LRO. Moreover, even if the exact pattern is known, one should check for all its symmetry-equivalent variations, since the value of $S_{\mathrm{A}}^{(\mathrm{PAT})}$ clearly depends on assumed orientation of PAT. Therefore, in principle, all variants of PAT produced by symmetry operations should be checked, as the variant developed during simulation is usually a result of random fluctuations and can not be predicted a priori. However, it would be very useful to have a quick way of checking whether long-range order of some type develops during Monte Carlo simulation or not, preferably without the $a$ priori knowledge about ordering pattern. 
It turns out that, in order to detect the presence of long-range ordering, quantities derived from $S_{\mathrm{A}}^{(\mathrm{PAT})}$ are more useful. Instead of a priori selecting ordered configuration PAT, it is helpful to calculate the Bragg-Williams parameter with one of the structures obtained in the developed stage of simulation used as pattern PAT. It is important that this reference structure PAT is selected far after the thermalization phase. The quantity calculated with this method we will call the simulation LRO parameter (sim-LRO) and denote it as $S_{\mathrm{A}}$. The typical behavior patterns of $S_{\mathrm{A}}$ during Monte Carlo simulation run are displayed in Fig. 3. They are taken from calculations for $\mathrm{Ga}_{x} \operatorname{In}_{1-x} \mathrm{~N}$ on InN substrate, which are discussed in greater detail in Sec. VI It turns out that three types of behavior for $S_{\mathrm{A}}$ can be distinguished. First, if no LRO is present then $S_{\mathrm{A}}$ simply fluctuates around 0 value, corresponding to unordered alloy. This is illustrated in Fig. 3 (a). Second, if certain LRO pattern was developed during simulation, the successive structures fluctuate around it generating values of $S_{\mathrm{A}}$ that are considerably different from 0 . This is depicted in Fig. 3 (b). Third, sometimes different arrangements of atoms could repeat during simulations, e.g., related to different orientation or shift of LRO pattern. It will correspond to series of peaks visible in $S_{\mathrm{A}}$ values during simulation time, see Fig. 3 (c). These peaks correspond to moments when the structure is similar to the one selected for comparison. Even though, the average values of $S_{\mathrm{A}}$ throughout the whole simulation could be in this case close to 0 , this behavior also indicates the development of LRO or precipitation. Therefore, to detect this type of ordering, except the average value of $S_{\mathrm{A}}$ we also analyze the spread of observed $S_{\mathrm{A}}$ values. Convenient measure here is the difference of percentiles $q$ related to 5 and 95 percent, estimated on the basis of $S_{\mathrm{A}}$ population generated during Monte Carlo (MC) sampling

$$
\Delta S_{\mathrm{A}}=q_{0.95}\left(S_{\mathrm{A}}\right)-q_{0.05}\left(S_{\mathrm{A}}\right) .
$$

This quantity provides the interval width which includes $90 \%$ of the observed data. The occurrence of high values for either average sim-LRO parameter $S_{\mathrm{A}}$ or its spread $\Delta S_{\mathrm{A}}$, enables for convenient detection of long range ordering in $\mathrm{MC}$ simulation results. The average value of $S_{\mathrm{A}}$ together with the percentiles forming $\Delta S_{\mathrm{A}}$ are marked with dashed lines in Figs. 3 (a)-(c).

\section{ORDERING IN THE TERNARY BULK ALLOYS}

In this section, the ordering in the bulk nitride ternary materials $\mathrm{Ga}_{x} \mathrm{In}_{1-x} \mathrm{~N}, \mathrm{Al}_{x} \mathrm{In}_{1-x} \mathrm{~N}$, and $\mathrm{Al}_{x} \mathrm{Ga}_{1-x} \mathrm{~N}$ is examined in a great detail. In the analyzed cases, no longrange ordering was observed. The sim-LRO parameter for all presented cases fluctuated around zero, similarly to the situation displayed in Fig. 3 (a). Therefore, we focus here on the short-range ordering. The Warren-Cowley SRO parameters for the first four coordination shells in

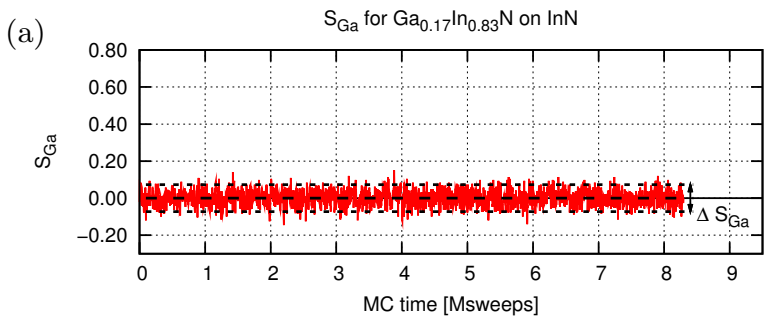

(b)
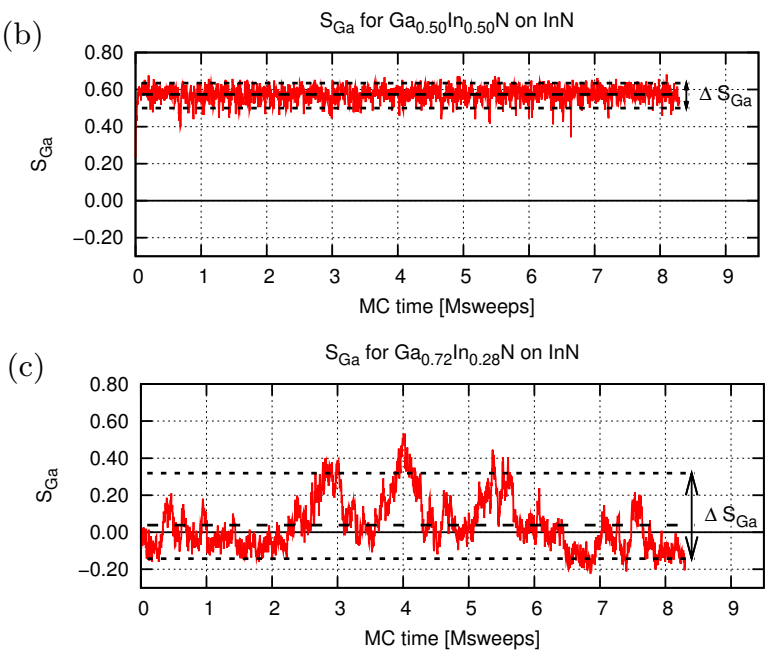

FIG. 3. (Color online) Different types of behavior of $S_{\mathrm{Ga}}$ during simulations for for $\mathrm{Ga}_{x} \operatorname{In}_{1-x} \mathrm{~N}$ alloy on InN substrate. Three gallium concentrations $x$ are presented: $x=0.17$ (a), $x=0.50$ (b), $x=0.72$ (c). Dashed lines correspond to the average value of $S_{\mathrm{Ga}}$ and its spread $\Delta S_{\mathrm{Ga}}$.

the cationic lattice are examined in the whole concentration range. Moreover, this study covers a few different temperatures, beginning roughly from the lower end of growth temperatures range, i.e., $873 \mathrm{~K}$, and including higher values up to $1673 \mathrm{~K}$. This upper limit could correspond to annealing during the post-processing phase. In the following the results for each of the ternary combinations, namely, $\mathrm{Ga}_{x} \mathrm{In}_{1-x} \mathrm{~N}, \mathrm{Al}_{x} \mathrm{In}_{1-x} \mathrm{~N}$, and $\mathrm{Al}_{x} \mathrm{Ga}_{1-x} \mathrm{~N}$, are described.

\section{A. Ordering in $\mathbf{G a}_{x} \operatorname{In}_{1-x} \mathbf{N}$}

$\mathrm{Ga}_{x} \operatorname{In}_{1-x} \mathrm{~N}$ is up to now the most investigated of all ternary combinations of nitrides. Currently, it is the main ingredient of the active region in blue-green optoelectronic devices. The dependencies of $\Gamma_{\text {GaIn }}^{(1)}, \Gamma_{\text {GaIn }}^{(2)}$, $\Gamma_{\text {GaIn }}^{(3)}$, and $\Gamma_{\text {GaIn }}^{(4)}$ on composition for different temperatures are presented in Fig. 4. The most interesting composition range, corresponding to violet-blue-green wavelength $400-570 \mathrm{~nm}$ is gray shaded on all graphs. It is easy to observe that $\Gamma_{\text {GaIn }}^{(1)}$ and $\Gamma_{\text {GaIn }}^{(4)}$ are negative in the whole concentration range and for all examined temperatures. This indicates the preference toward Ga-In neighboring on the first and fourth coordination shells. 
Conversely, $\Gamma_{\text {GaIn }}^{(2)}$ and $\Gamma_{\text {GaIn }}^{(3)}$ remain positive, which indicates that Ga atom is more likely to have another Ga atom on its second and third coordination shell, than it would result from concentration $x$. This sign pattern is in qualitative agreement with recent results of Chan and coworkers ${ }^{33}$ obtained on the basis of the cluster expansion model. This $-/+/+/-$ sequence observed for the $\Gamma^{(i)}$ parameters is usually interpreted as manifestation of stability for the chalcopyrite structure, which exhibits the same SRO signs pattern $\underline{33 / 45}$

For each dependence of the short-range order parameter on gallium concentration $x$, the third order polynomial was fitted. This type of curve is selected as the simplest model capable of describing observed shapes with asymmetric maximum/minimum. These fits are presented as continuous lines in Fig. 4. It turns out that all short-range order parameters dependencies have single extremum, corresponding to maximum absolute value of $\Gamma$ parameter. On the basis of obtained polynomial fits, concentrations $x_{\text {ext }}^{(i)}$ corresponding to this maximum ordering are determined. These extrema are marked with open symbols in Fig. 4. The numerical values of obtained $x_{\text {ext }}^{(i)}$ are presented in Table I. For the nearest neighbors and next nearest neighbors, the $x_{\text {ext }}^{(i)}$ is located at lower gallium concentration roughly around $35 \%$, or in other words at high indium content $65 \%$. These compositions are outside of the most interesting violet-blue-green range. For the third and fourth coordination shells, these extrema move toward approximately $50 \%$ compositions. Also the overall curve shape gets more symmetric around extremum for the case of $\Gamma_{\text {GaIn }}^{(3)}$ and $\Gamma_{\text {GaIn }}^{(4)}$ than it is in the case of the first and second shell. Nevertheless, these higher-order parameters should have weaker influence, e.g., on electronic properties of $\mathrm{Ga}_{x} \mathrm{In}_{1-x} \mathrm{~N}$ than $\Gamma_{\mathrm{GaIn}}^{(1)}$ and $\Gamma_{\mathrm{GaIn}}^{(2)}$. Therefore, we conclude that the region, where effects related to ordering are the most pronounced is located around $x \approx 0.35$. It is also worth noting that the position of $x_{\text {ext }}^{(i)}$ is virtually independent on temperature, and the difference

$$
\Delta x_{\mathrm{ext}}^{(i)}=x_{\mathrm{ext}}^{(i)}(T=1673 K)-x_{\mathrm{ext}}^{(i)}(T=873 K)
$$

for all coordination shells $i=1, \ldots 4$ does not exceed 0.04 . We also calculate the difference of extremum values for each $\Gamma^{(i)}$

$$
\Delta \Gamma_{\text {ext }}^{(i)}=\Gamma_{\text {ext }}^{(i)}(T=1673 K)-\Gamma_{\text {ext }}^{(i)}(T=873 K) .
$$

This quantity indicates that the ordering decreases roughly by half when the temperature changes from $T=873$ to $T=1673 \mathrm{~K}$. All the above findings are summarized in Table I.

As far as modeling of electronic structure for $\mathrm{Ga}_{x} \mathrm{In}_{1-x} \mathrm{~N}$ is concerned, the above results indicate that it would be the most interesting to focus on structures with $\Gamma_{\text {GaIn }}^{(i)}$ having successive signs $-/+/+/-$ as an input to density functional theory, tight-binding or empirical pseudopotential schemes. Methods which assume
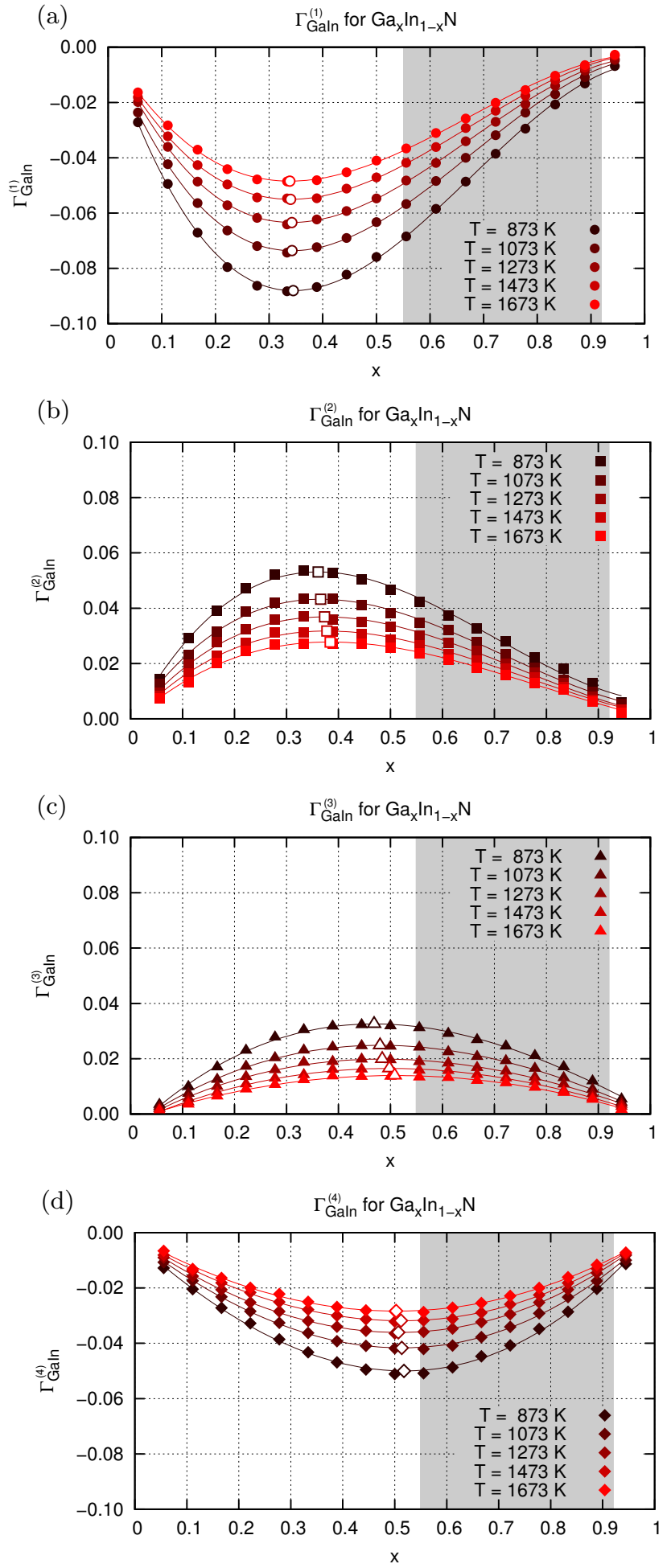

FIG. 4. (Color online) The short-range order parameters in $\mathrm{Ga}_{x} \operatorname{In}_{1-x} \mathrm{~N}$ as a function of composition for different temperatures. Note that the scale on all graphs is the same to allow for direct comparison of ordering magnitude in different coordination shells. Continuous lines represent third order polynomial fits to simulated data. The extrema for presented fits are marked with open symbols. The composition range approximately corresponding to violet-blue-green wavelength of emitted light $(400-570 \mathrm{~nm})$ is gray shaded. 
$\Gamma_{\mathrm{GaIn}}^{(i)}=0$ such as, e.g., special quasirandom structures (SQS) or virtual crystal approximation (VCA) could lead to systematic inaccuracy, caused by neglecting of the SRO. The range, where these inaccuracies should be the most pronounced, is determined by extrema for the lowest coordination shells SRO parameter, namely around indium concentration of $65 \%$ or so. On the other hand, when considering alloys corresponding to the violet end of spectra, say around $10 \%$ of indium, the influence of ordering effects should be much less pronounced. The comparison of the above results with experiment is not straightforward. There is a heated debate about the range of concentrations and, more generally, conditions that lead to In clustering in $\mathrm{Ga}_{x} \operatorname{In}_{1-x} \mathrm{~N}$ samples. This issue so far is by no means clarified, even on the phenomenological level. Our computations, to a certain extent, support these findings that report no clustering or long-range ordering in the samples even with high In concentration. [5] In our simulations we show that under the lattice-coherent assumption, thermodynamics does not prohibit obtaining mixtures without precipitates or LRO in very broad indium concentration range and in temperatures higher or in the region of the typical growth conditions. However, one has to stress the fact, that this state could be difficult to obtain experimentally, mostly due to artifacts related to epitaxial growth methods. These methods, such as MBE or metalorganic vapor phase epitaxy (MOVPE), take place on the surface, in conditions at least partially corresponding to non-equilibrium. Therefore, some features specific to growth process could form metastable state and remain "quenched" in the samples. To fully describe these phenomena, the detailed growth models should be developed. This is, however, far more difficult than already not-easy computation of equilibrium properties. Yet another issue not addressed on this stage of modeling is the presence of strain, both local and global. By local strain we mean deformations related to defects, in particular dislocations, whereas global strain occurs in thin lay-

TABLE I. The summary of extrema for $\Gamma(x)$ dependencies in $\mathrm{Ga}_{x} \mathrm{In}_{1-x} \mathrm{~N}$ presented in Fig. 4. Concentrations of maximum ordering $x_{\text {ext }}^{(i)}$, together with corresponding extremum value of $\Gamma_{\text {GaIn }}^{(i)}$ at the lowest examined temperature $T=873 \mathrm{~K}$ are provided. The influence of temperature is illustrated using the difference $\Delta x_{\text {ext }}^{(i)}$ between extremum concentrations at $T=$ $1673 \mathrm{~K}$ and $T=873 \mathrm{~K}$ [see Eq. (7)] and extremum values of SRO parameters $\Delta \Gamma_{\text {ext }}^{(i)}$ [see Eq. (8)].

\begin{tabular}{lcccr}
\hline \hline & $x_{\text {ext }}^{(i)}$ & $\Gamma_{\text {ext }}^{(i)}(\mathrm{T}=873 \mathrm{~K})$ & $\Delta x_{\mathrm{ext}}^{(i)}(\mathrm{T}=873 \mathrm{~K})$ & $\Delta \Gamma_{\mathrm{ext}}^{(i)}$ \\
\hline$\Gamma_{\mathrm{GaIn}}^{(1)}$ & 0.35 & -0.088 & -0.006 & 0.040 \\
$\Gamma_{\mathrm{GaIn}}^{(2)}$ & 0.36 & 0.053 & 0.023 & -0.025 \\
$\Gamma_{\mathrm{GaIn}}^{(3)}$ & 0.47 & 0.033 & 0.040 & -0.019 \\
$\Gamma_{\mathrm{GaIn}}^{(4)}$ & 0.52 & -0.050 & -0.015 & 0.022 \\
\hline \hline
\end{tabular}

ers and is related to lattice mismatch between substrate and alloy material. We reference it as global, since it is present in the whole volume of the considered layer. The influence of dislocations and other sources of local strain is not included in our modeling, since we assume perfect crystalline lattice. Taking into account such extended defects would require calculations with much larger supercells with complicated geometry, prohibitive for the detailed composition and temperature scans provided in this study. Nevertheless, we can gain certain insight into the influence of strain on ordering by modeling global epitaxial deformation. It was already indicated in the literature that this epitaxial strain can have significant impact on ordering phenomena. $22|23| 28|31| 34 \mid$ Therefore, its role is analyzed in detail in Sec. VI.

\section{B. Ordering in $\mathbf{A l}_{x} \operatorname{In}_{1-x} \mathbf{N}$}

When speaking of ternary nitride alloys with application prospects, $\mathrm{Ga}_{x} \mathrm{In}_{1-x} \mathrm{~N}$ is not the only option. Recently, also $\mathrm{Al}_{x} \operatorname{In}_{1-x} \mathrm{~N}$ attracts considerable attention. It is particularly interesting, because by adjusting the In composition one can lattice match it to GaN substrate, producing in principle strain-free interfaces and heterojunctions. The absence of strain has many advantages such as lack of electric field component related to piezoelectric effect, lower density of defects on the interface, etc. Therefore, such $\mathrm{Al}_{x} \operatorname{In}_{1-x} \mathrm{~N}$ layers are promising candidates for applications in optoelectronic devices, e.g., for high quality factor microcavities or distributed Bragg reflectors. Moreover, the AlInN/GaN junction could be an important building block for high electron mobility transistors (HEMTs). For more detailed information about properties and applications of $\mathrm{Al}_{x} \mathrm{In}_{1-x} \mathrm{~N}$, see a recent review paper by Butté and co-workers.

In our study of ordering in the ternary $\mathrm{Al}_{x} \operatorname{In}_{1-x} \mathrm{~N}$, we observe qualitatively similar behavior as in the case of $\mathrm{Ga}_{x} \operatorname{In}_{1-x} \mathrm{~N}$ (compare Figs. 4 and 5). Again, the same alternating pattern of signs for order parameters is observed, i.e., $\Gamma_{\text {AlIn }}^{(1)}$ and $\Gamma_{\text {AlIn }}^{(4)}$ are negative, whereas $\Gamma_{\text {AlIn }}^{(2)}$ and $\Gamma_{\text {AlIn }}^{(3)}$ are positive. Similarly to the previous section, the SRO parameter values and concentrations for extremum ordering $\Gamma_{\mathrm{ext}}^{(i)}$ and $x_{\mathrm{ext}}^{(i)}$ are determined. The extremal values of order parameters were in the case of $\mathrm{Al}_{x} \mathrm{In}_{1-x} \mathrm{~N}$ larger than in the case of $\mathrm{Ga}_{x} \operatorname{In}_{1-x} \mathrm{~N}$ roughly by a factor of $50 \%$, which correlates with larger lattice mismatch between AlN and InN than between the GaN and InN. For detailed comparison, see Table [I] and the analogous data for $\mathrm{Ga}_{x} \mathrm{In}_{1-x} \mathrm{~N}$ gathered in Table I] Important difference in the case of $\mathrm{Al}_{x} \operatorname{In}_{1-x} \mathrm{~N}$ is that the composition dependencies of $\Gamma_{\text {AlIn }}^{(2)}, \quad \Gamma_{\text {AlIn }}^{(3)}$, and $\Gamma_{\text {AlIn }}^{(4)}$ exhibit features of bimodal shape, particularly in lower temperatures. Therefore, in these cases the third-order polynomial fits carried out in order to locate extrema, were performed in the narrowed range: $x \in[0.0,0.6]$ for $\Gamma_{\text {AlIn }}^{(2)}, \quad \Gamma_{\text {AlIn }}^{(3)}$ and $x \in[0.0,0.7]$ for $\Gamma_{\text {AlIn }}^{(4)}$ The bimodal 
TABLE II. The summary of extrema for $\Gamma(x)$ dependencies in $\mathrm{Al}_{x} \operatorname{In}_{1-x} \mathrm{~N}$ presented in Fig. 5 . Concentrations of maximum ordering $x_{\text {ext }}^{(i)}$, together with corresponding extremum value of $\Gamma_{\text {AlIn }}^{(i)}$ at the lowest examined temperature $T=873 \mathrm{~K}$ are provided. Since in $\mathrm{Al}_{x} \operatorname{In}_{1-x} \mathrm{~N}$, for $\Gamma_{\text {AlIn }}^{(2)}$ and $\Gamma_{\text {AlIn }}^{(3)}$ two extrema are present, both of these are quoted in the table. The influence of temperature is illustrated using the difference $\Delta x_{\text {ext }}^{(i)}$ between extremum concentrations at $T=1673 \mathrm{~K}$ and $T=873 \mathrm{~K}$, [see Eq. (7)] and extremum values of SRO parameters $\Delta \Gamma_{\text {ext }}^{(i)}$ [see Eq. (8)].

\begin{tabular}{lcccr}
\hline \hline & $x_{\text {ext }}^{(i)}$ & $\Gamma_{\text {ext }}^{(i)}(\mathrm{T}=873 \mathrm{~K})$ & $\Delta x_{\text {ext }}(\mathrm{T}=873 \mathrm{~K})$ & $\Delta \Gamma_{\text {ext }}^{(i)}$ \\
\hline$\Gamma_{\text {AlIn }}^{(1)}$ & 0.31 & -0.131 & 0.006 & 0.055 \\
$\Gamma_{\text {Alln }}^{(2)}$ & 0.31 & 0.090 & 0.024 & -0.042 \\
& 0.70 & 0.067 & & \\
$\Gamma_{\text {Alln }}^{(3)}$ & 0.41 & 0.058 & 0.033 & -0.034 \\
& 0.66 & 0.052 & & \\
$\Gamma_{\text {AlIn }}^{(4)}$ & 0.50 & -0.076 & 0.016 & 0.033 \\
\hline \hline
\end{tabular}

character is particularly pronounced for $\Gamma_{\mathrm{AlIn}}^{(2)}, \Gamma_{\mathrm{AlIn}}^{(3)}$ in the lowest temperature $T=873 \mathrm{~K}$. In these cases, the second maxima are clearly visible and could be easily calculated from polynomial fits. The detailed information about all extrema is provided in Table II Apart from the bimodal character, other features observed for the SRO parameters in $\mathrm{Al}_{x} \mathrm{In}_{1-x} \mathrm{~N}$ are similar to $\mathrm{Ga}_{x} \mathrm{In}_{1-x} \mathrm{~N}$. The shape of extremum for $\Gamma_{\text {AlIn }}^{(1)}$ and $\Gamma_{\text {AlIn }}^{(2)}$ is asymmetric and located in the high indium concentration range around $70 \%$, a little bit higher than for the $\mathrm{Ga}_{x} \mathrm{In}_{1-x} \mathrm{~N}$ case. For the highest coordination shells, the extremum gets more symmetric and shifts toward $50 \%$ composition. Again for this alloy, the location of extremum concentration weakly depends on temperature and changes maximally around $3 \%$, as presented in Table II.

Since the properties of $\mathrm{Al}_{x} \operatorname{In}_{1-x} \mathrm{~N}$ lattice matched to $\mathrm{GaN}$ are of high practical importance, we gather the information about the short-range order parameters of this material in Table III In the context of wurtzite $\mathrm{Al}_{x} \operatorname{In}_{1-x} \mathrm{~N}$, usually the aluminum concentration $x \approx 0.82$ is quoted as corresponding to lattice matching. ${ }^{46}$ In the case of cubic materials and lattice parameters employed in this study, the Vegard's law leads to the concentration $x \approx 0.79$. For simplicity, we neglect here the small unphysical lattice bowing effect which is a feature of the Keating model $!^{42}$ The composition of the $\mathrm{Al}_{x} \mathrm{In}_{1-x} \mathrm{~N}$ lattice matching $\mathrm{GaN}$, is also marked for convenience in Fig. 5. It turns out that the magnitude of SRO parameters in this case does not exceed 0.05. Interestingly, it is the lowest for the first coordination shell, which should have the greatest impact on the alloy properties. When it comes to modeling implications, the situation here is analogous to $\mathrm{Ga}_{x} \mathrm{In}_{1-x} \mathrm{~N}$. Our calculations indicate that for electronic structure modeling the configuration with
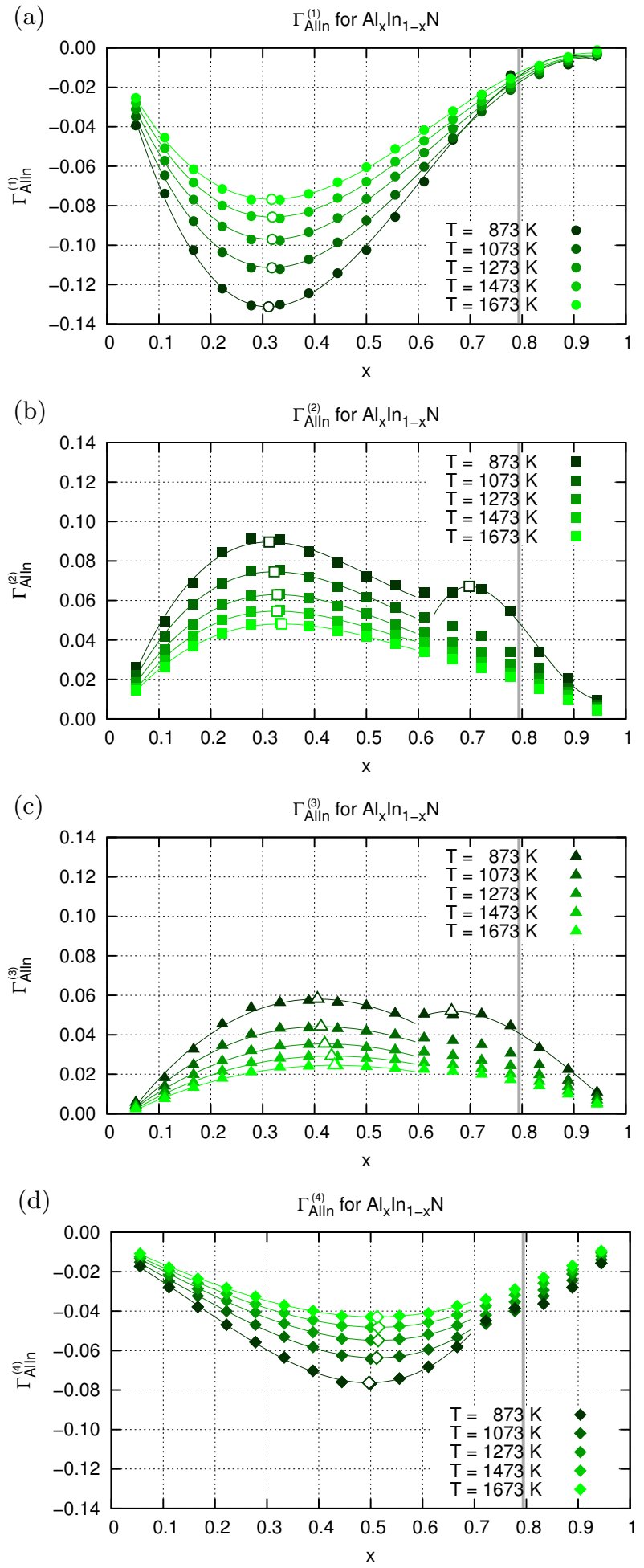

FIG. 5. (Color online) The short-range order parameters in $\mathrm{Al}_{x} \operatorname{In}_{1-x} \mathrm{~N}$ as a function of composition for different temperatures. Note that the scale on all graphs is the same to allow for direct comparison of ordering magnitude in different coordination shells. Continuous lines represent third order polynomial fits to simulated data. The extrema for presented fits are marked with open symbols. The composition of $\mathrm{Al}_{x} \mathrm{In}_{1-x} \mathrm{~N}$ lattice matched to $\mathrm{GaN}$ is indicated with gray line. 
SRO sign pattern $-/+/+/-$ should be the most interesting. The deviation from random uncorrelated alloy is the biggest for high indium concentration around $70 \%$, therefore, in this region models assuming $\mathrm{SRO}=0$ should have the largest systematic inaccuracy.

TABLE III. Summary of the short-range order parameters for $\mathrm{Al}_{x} \operatorname{In}_{1-x} \mathrm{~N}$ lattice matched to $\mathrm{GaN}$, i.e., for aluminum concentration $x \approx 0.79$.

\begin{tabular}{lrr}
\hline \hline & $\mathrm{T}=873 \mathrm{~K}$ & $\mathrm{~T}=1673 \mathrm{~K}$ \\
\hline$\Gamma_{\text {AlIn }}^{(1)}$ & -0.014 & -0.012 \\
$\Gamma_{\text {AlIn }}^{(2)}$ & 0.049 & 0.019 \\
$\Gamma_{\text {AlIn }}^{(3)}$ & 0.041 & 0.016 \\
$\Gamma_{\text {AlIn }}^{(4)}$ & -0.038 & -0.027 \\
\hline \hline
\end{tabular}

\section{Ordering in $\mathbf{A l}_{x} \mathbf{G a}_{1-x} \mathbf{N}$}

Finally, the last possible ternary combination for the examined family of nitrides is $\mathrm{Al}_{x} \mathrm{Ga}_{1-x} \mathrm{~N}$. This material is very well suited for high electron mobility transistors, operating in high-power range as well as ultraviolet light emitters and detectors. It is also a promising building block for biosensors. Since the lattice mismatch and the differences in force field parameters are the smallest in this case, also the ordering effects here are the weakest. For this material, we carried out the studies only for two temperatures 873 and $1673 \mathrm{~K}$. None of the examined $\Gamma_{\mathrm{AlGa}}^{(i)}$ values exceeded 0.01 . Therefore, our conclusion is that $\mathrm{Al}_{x} \mathrm{Ga}_{1-x} \mathrm{~N}$ follows quite closely the picture of of uncorrelated random alloy, i.e., with both $\mathrm{SRO}=0$ and $\mathrm{LRO}=0$.

\section{ORDERING IN TERNARY ALLOYS ON THE SUBSTRATE}

In the previous section we have examined ordering phenomena in bulk crystals. However, for applications in optoelectronic devices or sensors usually thin epitaxial layers grown on substrate are employed. In this part, we describe, how the presence of substrate associated with biaxial strain influences the ordering phenomena. We focus on the technologically most important case of $\mathrm{Ga}_{x} \operatorname{In}_{1-x} \mathrm{~N}$ and examine its behavior on a variety of substrates. However, before moving on to the discussion of ordering, the basic facts about thin epitaxial layers are summarized below.

The main parameter for epitaxial layer grown on the substrate is misfit strain. For a cubic material, it is defined as

$$
\epsilon_{\text {misfit }}=\frac{a_{\text {substrate }}-a_{\text {layer }}}{a_{\text {layer }}}
$$

where $a_{\text {substrate }}$ and $a_{\text {layer }}$ denote substrate and layer alloy lattice constants respectively. If we consider the simplest model, the misfit strain is compensated by purely elastic deformation of the thin film material. The thick substrate is assumed to stay undeformed in this approach. See Fig. 6 for illustration of such a situation. Within this model, the deformation of layer unit cell on the interface (in order to match the substrate lattice) is compensated by relaxation in the perpendicular direction. One can approximate the relaxation strain $\epsilon_{\text {relax }}$ associated with accommodation of misfit strain using linear elasticity theory

$$
\epsilon_{\text {relax }}=-2 \frac{c_{12}}{c_{11}} \epsilon_{\text {misfit }}
$$

However, for large misfit strains going beyond the applicability range of the linear elasticity (few percent deformations), this expression can be a rather rough approximation. It is important to note that in the case

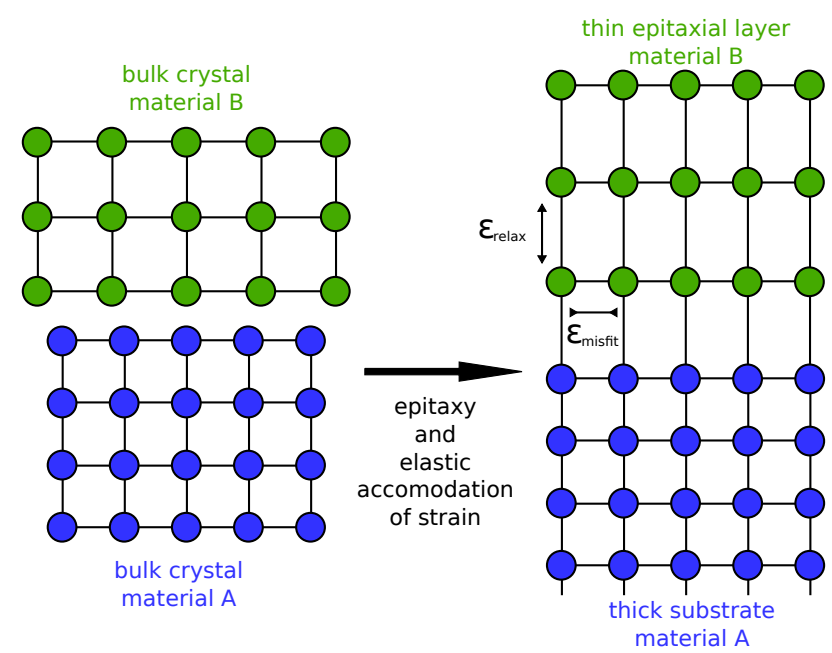

FIG. 6. (Color online) The epitaxial layer in the presence of lattice mismatch between the layer material and the substrate material. The case of purely elastic accommodation of strain is presented, i.e., without misfit dislocations (lattice-coherent case).

of lattice mismatch between the substrate and epitaxial, material there exists a critical thickness $h$, beyond which layer starts to relax the strain in the form of misfit dislocations and other defects, instead of purely elastic deformation. This means that because of the high defects density, epitaxial layers beyond $h$ are virtually useless from the point of view of light-emitting heterostructures. However, estimation of the critical thickness is a difficult problem. Various models were proposed for this purpose. In this paper, in order to get a rough estimate of the critical thickness, we employ a simple approach developed by Matthews and Blakeslee ${ }^{47}$ and later on employed to cubic nitrides ${ }^{48}$ It predicts that $h$ is given by the transcenden- 
tal equation

$$
h=\frac{a_{\text {substrate }}(1-\nu / 4)}{4 \sqrt{2} \pi(1+\nu)\left|\epsilon_{\text {misfit }}\right|}\left[\ln \left(\frac{\sqrt{2} h}{a_{\text {substrate }}}\right)+1\right],
$$

where $\nu$ is the Poisson ratio of the layer material $\nu=$ $c_{12} /\left(c_{11}+c_{12}\right)$. Note that since both misfit strain and Poisson ratio depend on alloy composition, the resulting critical thickness also depends on the proportion of compounds in alloy epitaxial layer. Employed values of lattice constants are given in Table IV the composition dependencies of the elastic parameters in Eq. (11) were taken from our previous work ${ }^{42}$ and are given by

$$
\begin{aligned}
& c_{11}(x)=182.23+105.70 x-15.44 x(1-x), \\
& c_{12}(x)=104.78+25.02 x-1.00 x(1-x) .
\end{aligned}
$$

For certain compositions related to large misfit strains, the real-valued solutions of the Eq. 11 do not exist, which indicates that fabricating even very thin layers of high crystalline quality is impossible for certain alloy/substrate combinations. However, one has to bear in mind, that accurate modeling of the critical thickness is complicated task in itself. The Matthews and Blakeslee approach is simple, but crude model. Therefore, it gives more qualitative than quantitative picture of the $h$ dependence on composition and limits of the elastic strain accommodation regime.

In the present section we examine the $\mathrm{Ga}_{x} \operatorname{In}_{1-x} \mathrm{~N}$ ternary alloy grown on a large variety of possible substrates, both employed experimentally as well as possibly suitable in the future, including 3C-SiC, zb-ZnO, $\mathrm{CaO}$, AlN, GaN, and InN. Currently, the most promising well lattice matched substrates for the zinc blende nitrides seem to be $3 \mathrm{C}-\mathrm{SiC}$ or $\mathrm{GaN}$ obtained either in the form of thick buffer layer on a different substrate or recently fabricated zb-GaN free-standing crystals. $\stackrel{49}{51}$ However, with technological progress, other options can gain significance in the future. The information about misfits bounds for each of these materials is gathered in Table IV

To simplify the discussion, we separate our results into two groups of substrates related to moderate and large misfit strains, since the observed phenomena in both cases are quite different. The first group consists of $\mathrm{GaN}$, zb-ZnO, and $\mathrm{CaO}$, whereas the second comprises 3C-SiC, AlN, and InN. All the results have been obtained in $T=873 \mathrm{~K}$, corresponding to a typical growth temperature range.

\section{A. Moderate misfits regime}

For substrates related to moderate misfit strains (i.e., $\mathrm{GaN}$, zb-ZnO, and $\mathrm{CaO}$ ), no long-range ordering is observed in $\mathrm{Ga}_{x} \operatorname{In}_{1-x} \mathrm{~N}$, similarly to the bulk case. Since the intensity of ordering effects in materials is quantified by the absolute value of short-range order parameter
TABLE IV. Misfit strain range for various substrate applicable to $\mathrm{Ga}_{x} \operatorname{In}_{1-x} \mathrm{~N}$ alloys. The extremum values for pure GaN and pure $\mathrm{InN}$ are provided.

\begin{tabular}{cccc}
\hline \hline Substrate & $\begin{array}{c}\text { Lattice } \\
\text { constant }\end{array}$ & $\begin{array}{c}\text { Misfit strain range for } \begin{array}{c}\mathrm{Ga}_{x} \operatorname{In}_{1-x} \mathrm{~N} \\
x=1 \\
\text { (pure GaN) }\end{array} \\
\text { (pure InN) }\end{array}$ \\
\hline $3 \mathrm{C}-\mathrm{SiC}$ & 4.360 & $-3.2 \%$ & $-12.8 \%$ \\
$\mathrm{AlN}$ & 4.374 & $-2.9 \%$ & $-12.5 \%$ \\
$\mathrm{GaN}$ & 4.503 & $0.0 \%$ & $-9.9 \%$ \\
$\mathrm{zb}-\mathrm{ZnO}$ & 4.580 & $1.7 \%$ & $-8.4 \%$ \\
$\mathrm{CaO}$ & 4.811 & $6.8 \%$ & $-3.8 \%$ \\
$\mathrm{InN}$ & 5.000 & $11.0 \%$ & $0.0 \%$ \\
\hline \hline
\end{tabular}

$\left|\Gamma_{\mathrm{GaIn}}^{(i)}\right|$, it is useful to analyze the influence of the substrate in terms of $\Delta \Gamma_{\text {GaIn }}^{(i)}$ defined as

$$
\Delta \Gamma_{\text {GaIn }}^{(i)}=\mid \Gamma_{\text {GaIn }}^{(i)}(\text { on substrate })|-| \Gamma_{\text {GaIn }}^{(i)}(\text { bulk }) \mid .
$$

If the presence of the substrate does not change the sign of $\Gamma_{\mathrm{GaIn}}^{(i)}$ (which is the case for examined GaN, zb-ZnO, and $\mathrm{CaO})$, the interpretation of $\Delta \Gamma_{\mathrm{GaIn}}^{(i)}$ is straightforward. When $\Delta \Gamma_{\mathrm{GaIn}}^{(i)}>0$, the ordering effects are increased, whereas for $\Delta \Gamma_{\text {GaIn }}^{(i)}<0$, the grown thin layer behaves closer to the uncorrelated random alloy than the bulk case.

As an example of obtained results, the case of GaN substrate is presented in Fig. 7. The findings for $\mathrm{CaO}$ and $\mathrm{ZnO}$ are qualitatively similar. The graphs illustrate the comparison of the short-range order parameters $\Gamma_{\text {GaIn }}^{(1)}, \quad \Gamma_{\text {GaIn }}^{(2)}, \quad \Gamma_{\text {GaIn }}^{(3)}$, and $\Gamma_{\text {GaIn }}^{(4)}$ between strained and unstrained cases. Order parameters are accompanied by the corresponding $\Delta \Gamma_{\text {GaIn }}^{(i)}$ values. Also, the misfit strain $\epsilon_{\text {misfit }}$ and the relaxation strain $\epsilon_{\text {relax }}$ dependence on composition are provided for completeness. Finally, the information about the critical thickness $h$ calculated from the Matthews-Blakeslee model given by Eq. (11) is included. This provides insight, as to range of composition is of practical importance.

The $\epsilon_{\text {relax }}$ presented in Fig. 7 (c) has been calculated using two methods: first, within the theory of elasticity (TOE) and Eq. (10), second by relaxing the unit cell to the shape corresponding to minimum energy employing the Keating valence force field (VFF). For GaN and other examined substrates, both methods give similar results. The small differences emerged for compositions related to larger misfit strains. This is intuitively well understood, since the Keating model was constructed to recover the predictions of the TOE for small strains. W1 We also observed that for negative misfit strains (compression in the substrate plane), the theory of elasticity slightly overestimates the $\epsilon_{\text {relax }}$, whereas for positive misfits (in the case of zb- $\mathrm{ZnO}$ and $\mathrm{CaO}$ substrate), the $\epsilon_{\text {relax }}$ predicted by 
(a)

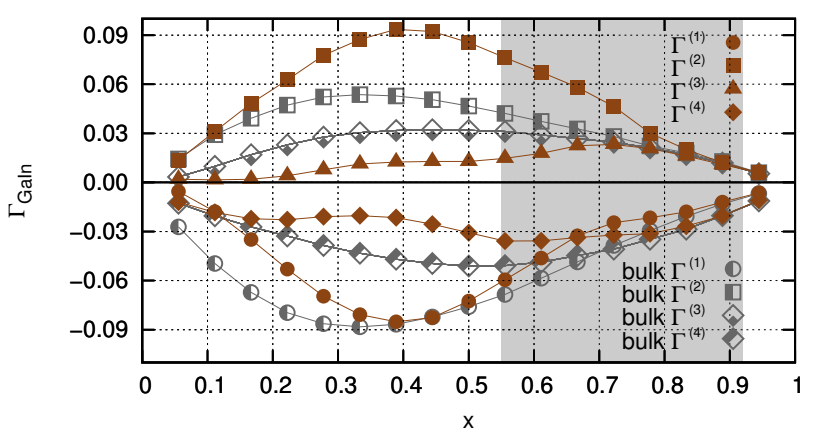

(b)

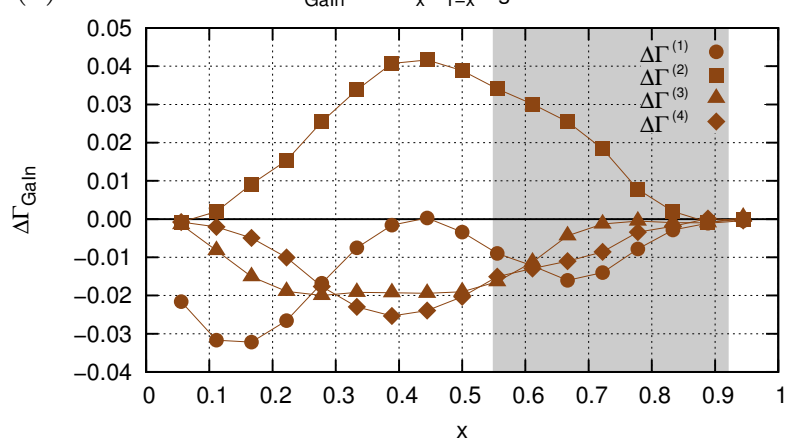

(c)

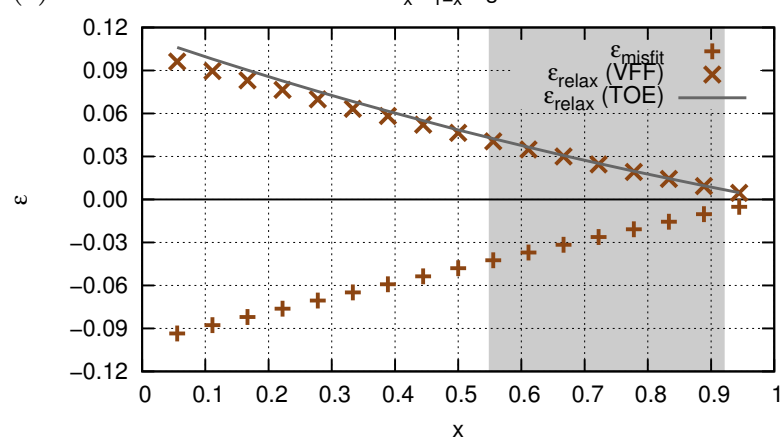

(d)

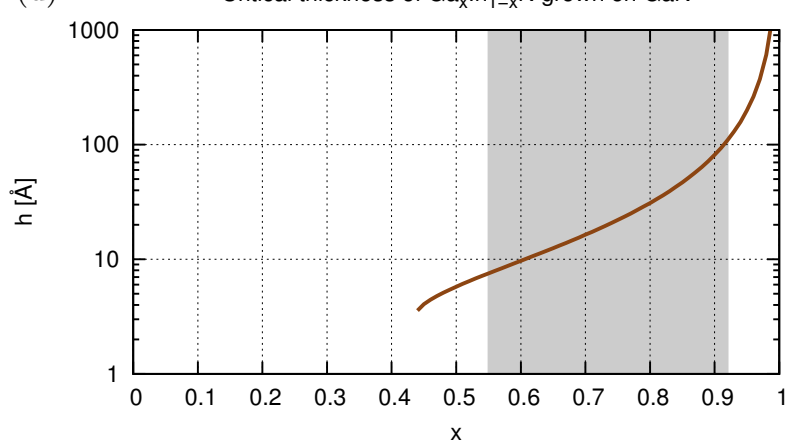

FIG. 7. (Color online) Summary of quantities related to ordering in $\mathrm{Ga}_{x} \operatorname{In}_{1-x} \mathrm{~N}$ grown on GaN. The SRO parameters $\Gamma_{\text {GaIn }}^{(i)}(\mathrm{a})$, their difference with respect to bulk case $\Delta \Gamma_{\text {GaIn }}^{(i)}$ (b), the misfit/relaxation strains calculated on the basis of elasticity theory (TOE) or Keating VFF (c), and the critical thickness (d). The composition range approximately corresponding to violet-blue-green wavelength of emitted light $(400-570 \mathrm{~nm})$ is gray shaded. the Keating force field is a little bit larger than calculated from elasticity. When it comes to the analysis of the substrate influence on order parameters, it can be clearly seen from Figs. 7 (a) and (b), that the most sensitive to the presence of substrate is $\Gamma_{\mathrm{GaIn}}^{(2)}$, as the magnitude of $\Delta \Gamma_{\mathrm{GaIn}}^{(2)}$ is the largest. Similar behavior is observed not only for the GaN, but also for zb-ZnO, and $\mathrm{CaO}$.

Interestingly, for both negative and positive misfits (present in the case of zb-ZnO and $\mathrm{CaO}$ ), the $\Delta \Gamma_{\mathrm{GaIn}}^{(2)}$ is positive, indicating that the presence of the substraterelated strain increases the ordering within this shell. On the other hand, the quantities $\Delta \Gamma_{\text {GaIn }}^{(1)}$ and $\Delta \Gamma_{\text {GaIn }}^{(4)}$ are mostly negative, showing the decreasing degree of ordering compared to the bulk case for these coordination shells. The magnitude of this effect is, however, lower than in the case of $\Delta \Gamma_{\text {GaIn }}^{(2)}$. Finally, the observed behavior of $\Delta \Gamma_{\text {GaIn }}^{(3)}$ is dependent on the sign of misfit strain. For the negative $\epsilon_{\text {misfit }}$, the decreased ordering is observed $\left(\Delta \Gamma_{\text {GaIn }}^{(3)}<0\right)$, and for positive values of the $\epsilon_{\text {misfit }}$, the ordering increases $\left(\Delta \Gamma_{\mathrm{GaIn}}^{(3)}>0\right)$.

Let us also mention that the previous calculations by Liu and Zunger using epitaxial cluster expansion 31 32 predict that already the influence of the GaN substrate triggers the phase separation and long-range ordering in $T=873 \mathrm{~K}$. They obtained the full miscibility in temperatures above $T_{M}=1080 \mathrm{~K}$. Our model predicts similar phenomena, however, in order to observe them substrates related to higher strains are necessary, as discussed in the next section.

\section{B. Large misfits regime}

In this section, we describe the behavior of $\mathrm{Ga}_{x} \operatorname{In}_{1-x} \mathrm{~N}$ on substrates that are related to larger strains, i.e., 3C-SiC, AlN, and InN. It turns out that in these cases the long-range ordering can be triggered. This is detected by analyzing the sim-LRO parameter $S_{\mathrm{Ga}}$ and its spread $\Delta S_{\mathrm{Ga}}$, as described in Sec. IV. For conditions where either $S_{\mathrm{Ga}}$ or $\Delta S_{\mathrm{Ga}}$ are considerably larger than in the bulk case, various ordered structures can be observed in the configurations generated during the Monte Carlo run. The details of formed patterns depend on composition as well as on the magnitude and sign of strain induced by the substrate. For the InN base layer, the $\mathrm{Ga}_{x} \mathrm{In}_{1-x} \mathrm{~N}$ alloy undergoes tensile strain, whereas for the $3 \mathrm{C}-\mathrm{SiC}$ and AlN the strain is compressive. Generally, both $3 \mathrm{C}-\mathrm{SiC}$ and AlN correspond to similar misfits as displayed in Table IV therefore, only the $3 \mathrm{C}-\mathrm{SiC}$ case will be presented in detail. The effect of the AlN substrate is analogous.

Let us begin with the analysis of the InN substrate. The complete set of characteristic quantities for this case is presented in Fig. 8. Graphs include the dependencies on composition of the long-range order parameter $S_{\mathrm{Ga}}$ and its spread $\Delta S_{\mathrm{Ga}}$, the short-range order parameters $\Gamma_{\mathrm{GaIn}}^{(i)}$, the relaxation together with misfit strains, and 

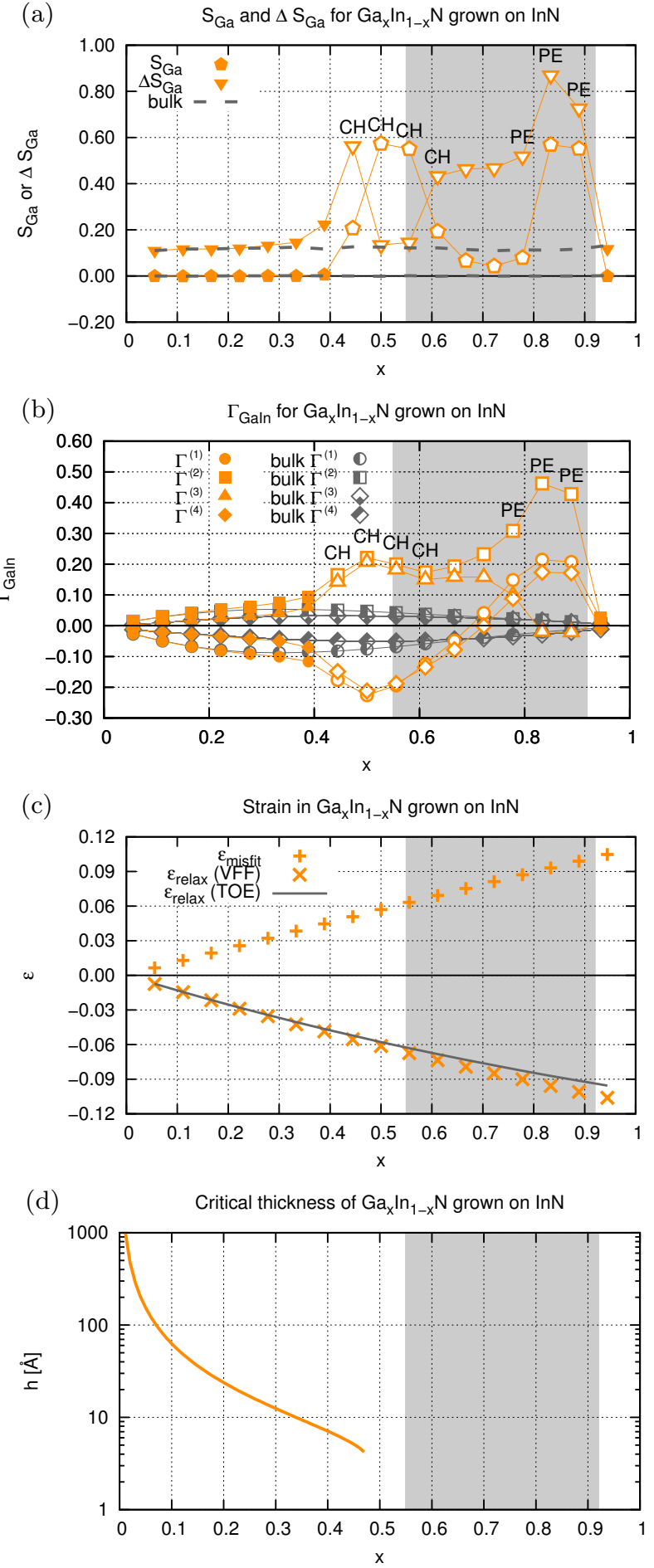

FIG. 8. (Color online) Summary of the quantities related to the ordering in $\mathrm{Ga}_{x} \mathrm{In}_{1-x} \mathrm{~N}$ grown on InN. The sim-LRO parameter $S_{\mathrm{Ga}}$ and its spread $\Delta S_{\mathrm{Ga}}$ (a), the SRO parameters $\Gamma_{\text {GaIn }}^{(i)}(\mathrm{b})$, the misfit/relaxation strains calculated on the basis of the elasticity theory (TOE) or the Keating VFF (c), and the critical thickness (d). Open symbols denote the occurrences of the LRO. CH stands for the chalcopyrite and PE for the perpendicular planes ordering. The composition range approximately corresponding to violet-blue-green wavelength of emitted light $(400-570 \mathrm{~nm})$ is gray shaded. (a)

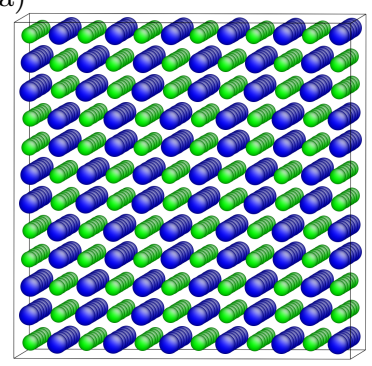

(b)

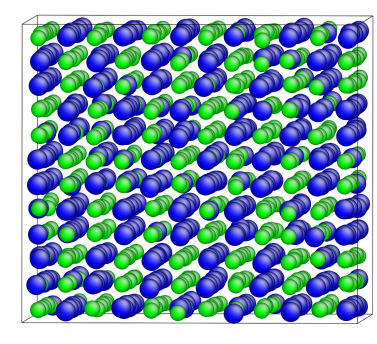

FIG. 9. (Color online) Atomic arrangement in the chalcopyrite structure (the $\mathrm{CH}$ pattern). The ideal structure (a) is compared with the sample atomic arrangement obtained for $\mathrm{Ga}_{0.5} \operatorname{In}_{0.5} \mathrm{~N}$ on InN during Monte Carlo simulation (b). Note that expansion in the basal (substrate) plane and corresponding compression in the perpendicular plane is visible, together with thermal displacements.

critical thickness. One can easily see that the sim-LRO parameter indicates the presence of the long-range ordering in the range $0.40<x<0.90$, since either the $S_{\mathrm{Ga}}$ or $\Delta S_{\mathrm{Ga}}$ reaches high values, compared to the bulk case. The observed dependencies of the LRO parameters on Monte Carlo time are in these cases similar to the sample series presented in Fig. 3 (b) and (c). The presence of the LRO in the range $0.40<x<0.90$ is also indicated by much higher values of the $\Gamma_{\mathrm{GaIn}}^{(i)}$ compared to the bulk case. It is worth noting that the whole violet-blue-green composition region lies completely in the discussed LRO regime for the considered InN substrate. The visual inspection of the structures obtained during simulations reveals that for concentrations around $x=0.5$ the so called chalcopyrite $(\mathrm{CH})$ pattern develops in the examined crystal. The ideal chalcopyrite structure is presented in Fig. 9 (a) and compared with one of the structures obtained during simulations in Fig. 9 (b). Note that even though both the deformation due to epitaxial strain and the effect of thermal vibrations are visible in the Monte Carlo structure, its similarity to the perfect chalcopyrite is clear. The concentrations corresponding to the chalcopyrite ordering pattern are marked in Fig. 8 with the $\mathrm{CH}$ label. It is well known from the literature that this type of ordering is energetically very favorable in the case of strained semiconductor alloys, not only nitrides $52 \sqrt[54]{54}$ Therefore, its appearance in our results is consistent with the previous findings. Moreover, the chalcopyrite pattern developed in our simulations is oriented perpendicularly to the substrate plane $\left(\mathrm{CH}_{\perp}\right)$ as predicted in the recent work of Liu and coworkers for large misfits. ${ }^{[54}$ The study of the remaining part of the concentration range reveals that the $\mathrm{CH}$ pattern is not the only ordering option. It turns out that different atomic arrangement occurs for the higher Ga concentration $x>0.7$. It consists of the In planes perpendicular to the substrate. The concentrations corresponding to this behavior are marked by label PE in Fig. 8. Sample structure with this type of ordering is presented in Fig. 11 (a). Such a behavior can be viewed as a certain mode of phase separation. 


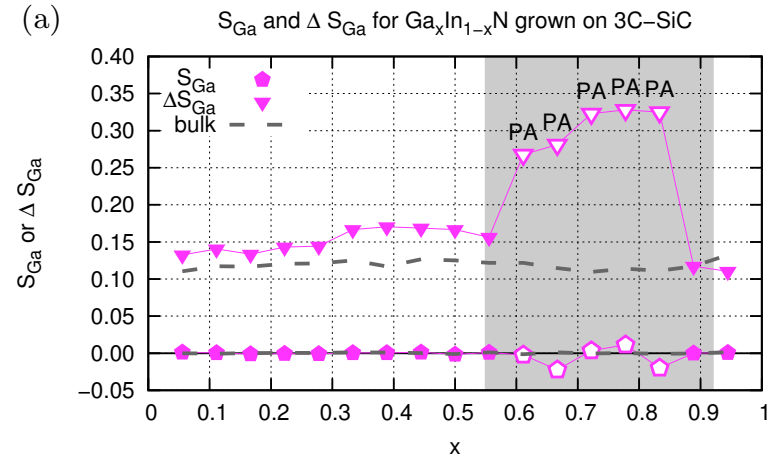

(b)
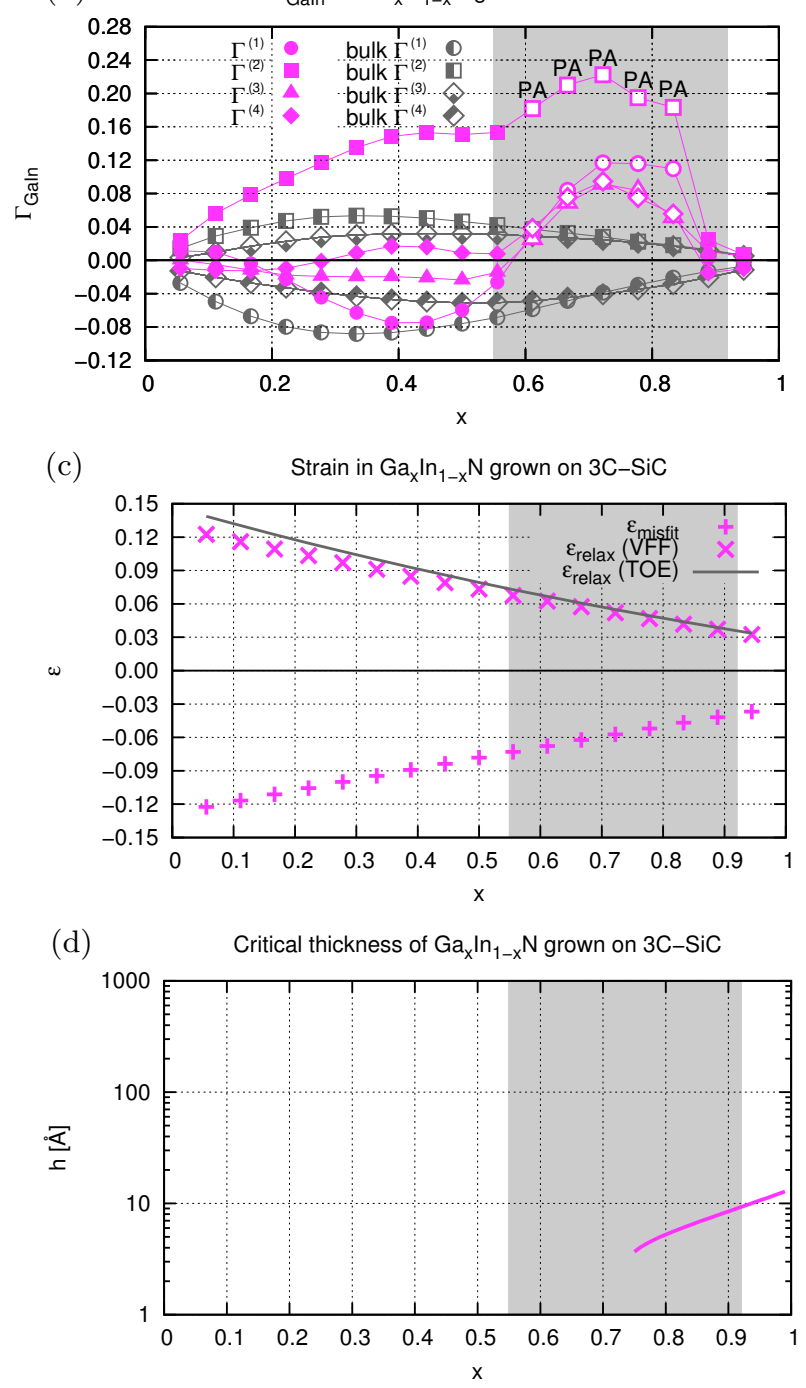

FIG. 10. (Color online) Summary of the quantities related to the ordering in $\mathrm{Ga}_{x} \mathrm{In}_{1-x} \mathrm{~N}$ grown on $3 \mathrm{C}-\mathrm{SiC}$. The sim-LRO parameter $S_{\mathrm{Ga}}$ and its spread $\Delta S_{\mathrm{Ga}}$ (a), the SRO parameters $\Gamma_{\text {GaIn }}^{(i)}(\mathrm{b})$, the misfit/relaxation strains calculated on the basis of the elasticity theory (TOE) or the Keating VFF (c), and the critical thickness (d). Open symbols denote the occurrences of the LRO of the parallel planes type (PA). The composition range approximately corresponding to violetblue-green wavelength of emitted light $(400-570 \mathrm{~nm})$ is gray shaded. (a)

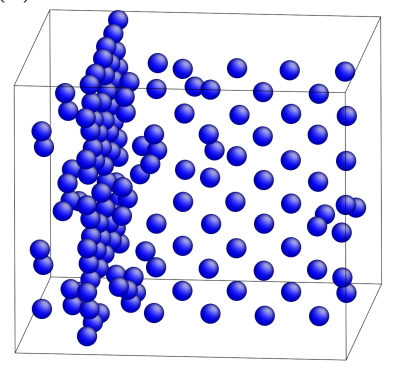

(b)

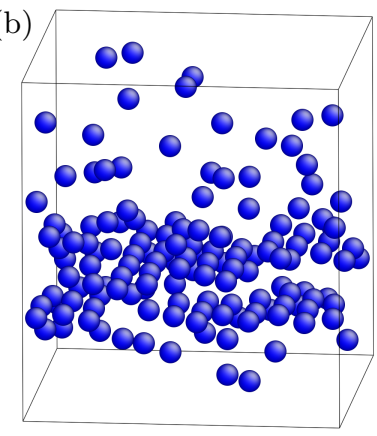

FIG. 11. (Color online) Different planar ordering observed in $\mathrm{Ga}_{0.83} \operatorname{In}_{0.17} \mathrm{~N}$ for two different substrates. For InN substrate, the ordering in the direction perpendicular to the substrate (the PE pattern) occurs (a). For SiC substrate, ordering in the planes parallel to the substrate (the PA pattern) is present (b). Only In atoms (minor component) are displayed for clarity.

This is also indicated by the fact that $\Gamma_{\mathrm{GaIn}}^{(1)}, \Gamma_{\mathrm{GaIn}}^{(2)}$, and $\Gamma_{\mathrm{GaIn}}^{(4)}$ shift toward high positive values, indicating the preference toward $\mathrm{Ga}-\mathrm{Ga}$ and $\mathrm{In}-\mathrm{In}$ neighboring. The structures without the $\mathrm{CH} / \mathrm{PE}$ symbols correspond to mixed ordering carrying certain features of both arrangements. The rest of the concentration range, namely for $x<0.4$ and for $x>0.9$, manifests no long range ordering, similarly to the case of $\mathrm{GaN}$, zb- $\mathrm{ZnO}$, and $\mathrm{CaO}$ substrates. It is also worth noticing that the $\mathrm{CH}$ ordering is observed close to the limit of elastic accommodation of strain regime, marked by the existence of critical thickness solutions in Eq. (11). The PE ordering in turn is observed well beyond this regime. Therefore, in reality the PE case might be difficult to observe due to the very poor crystalline layer quality in this misfit region.

The behavior of $\mathrm{Ga}_{x} \operatorname{In}_{1-x} \mathrm{~N}$ on substrates corresponding to large compressive strains also leads to the formation of the long-range ordering. The detailed dependencies on concentration for the long-range order characteristics $S_{\mathrm{Ga}}$ and $\Delta S_{\mathrm{Ga}}$, the short-range order parameters $\Gamma_{\mathrm{GaIn}}^{(i)}$, the relaxation and misfit strains accompanied by the critical thickness for $\mathrm{SiC}$ substrate are presented in Fig. 10. The analogous results for AlN are not included, since they were almost identical. From our simulations it emerges that for the high gallium concentrations in the range $0.60<x<0.85$ the long-range ordering can be observed. Even though $S_{\mathrm{Ga}}$ remains close to zero, the dependence of $\Delta S_{\mathrm{Ga}}$ on concentration experiences abrupt change in the ordering region. This change indicates that the $S_{\mathrm{Ga}}$ dependence on MC time is similar to the sample presented in Fig. 3 (c). This is also confirmed by the larger values of the short-range order parameters in this regime. It is worth noting that all $\Gamma_{\mathrm{GaIn}}^{(i)}$ become positive, indicating $\mathrm{Ga}-\mathrm{Ga}$ and $\mathrm{In}-\mathrm{In}$ neighboring preference. Visual inspection of configuration from the region with LRO reveals that indeed atoms tend to cluster in the planes parallel to the substrate. This is depicted in Fig. 10 as the PA pattern. Such a behavior can be inter- 
preted as a certain mode of phase separation. The sample structure corresponding to this arrangement is displayed in Fig. 11 (b). For the 3C-SiC substrate, this ordering occurs partially within the region of elastic strain accommodation marked by the existence of solutions for the critical thickness model from Eq. (11), so it should be possible to access it experimentally. Moreover, the whole discussed area of the LRO existence lies within the technologically important violet-blue-green composition region. For the Ga concentrations below the $x=0.6$, which correspond to the very high compressive strains, no significant indications of the long-range order are observed. Interestingly, the parameters $\Gamma_{\mathrm{GaIn}}^{(1)}, \Gamma_{\mathrm{GaIn}}^{(3)}$, and $\Gamma_{\mathrm{GaIn}}^{(4)}$ in this concentration region have absolute values lower than in the unstrained bulk case. The $\Gamma_{\text {GaIn }}^{(2)}$, in turn, similarly to the moderate strain case, deviates mostly from the bulk ordering and its absolute value increases. These phenomena, however, occur well outside the elastic accommodation of strain regime, where in reality extremely poor crystal quality would be obtained.

\section{SUMMARY}

In this paper, the ordering phenomena in ternary nitride alloys have been examined in a great detail. We have investigated how temperature, biaxial strain related to the presence of substrate, and the change of composition in the whole available range could influence type and degree of ordering. Our approach included vibrational contribution, which is not so common in the literature, where restricting to configurational degrees of freedom only is a frequent approximation. As a starting point bulk $\mathrm{Ga}_{x} \mathrm{In}_{1-x} \mathrm{~N}, \mathrm{Al}_{x} \mathrm{In}_{1-x} \mathrm{~N}$, and $\mathrm{Al}_{x} \mathrm{Ga}_{1-x} \mathrm{~N}$ have been investigated. The $\mathrm{Al}_{x} \mathrm{Ga}_{1-x} \mathrm{~N}$ has been found to follow closely uncorrelated random alloy picture, without both SRO and LRO. For the mixtures containing indium, no signs of long-range ordering or precipitation have been found in the bulk case; however, a considerable degree of short-range ordering has been observed. The short-range order parameters corresponding to the first four coordination shells followed the $-1+1+1-$ sign pattern, which agrees with the behavior observed in simulations for $\mathrm{Ga}_{x} \operatorname{In}_{1-x} \mathrm{~N}$ on the basis of cluster expansion model $[33$ Both materials deviate the strongest from the random uncorrelated alloy at high indium concentrations around $65 \%-70 \%$. This means that theoretical methods neglecting SRO should yield the largest systematic error in this concentration range. When it comes to resolving the In clustering controversy reported in the literature $\stackrel{5 / 7 / 9 \mid 10}{,}$ our findings could provide a certain support for the experimental results showing uniform distribution of In atoms in $\mathrm{Ga}_{x} \operatorname{In}_{1-x} \mathrm{~N}$ samples. However, the model of bulk alloy might not be fully appropriate here, since examined nitride alloys are usually employed in (opto)electronic devices in the form of strained epitaxial layers. Therefore, the next phase of our studies has been devoted to the influence of the substrate related biaxial strain on $\mathrm{Ga}_{x} \operatorname{In}_{1-x} \mathrm{~N}$ layers. A number of different substrates has been examined and classified into two groups. The first group, associated with moderate strains, contained $\mathrm{GaN}, \mathrm{CaO}$, and zb-ZnO. In this case, in the examined temperature $T=873 \mathrm{~K}$, still only $\mathrm{SRO}$ has occurred. The second and more interesting group comprised substrates yielding larger misfits, namely AlN, $3 \mathrm{C}-\mathrm{SiC}$, and InN. For these substrates, the appearance of the LRO has been detected by introduced sim-LRO parameter. For compressive misfit strain (AlN, 3C-SiC), the parallel planes (PA) pattern has developed for the In content $15 \%-40 \%$. For tensile misfit strain ( InN) the LRO regime has covered the region of $10 \%-60 \%$ of In content. The chalcopyrite ordering $(\mathrm{CH})$ has emerged, when the indium concentration was around 50\%, whereas for lower values down to $10 \%$, the perpendicular planes (PE) pattern has been found. The PA and the PE cases represent certain types of precipitation. The above results shed more light on the issue of ordering in nitrides occurring in thermodynamical equilibrium. We believe that the knowledge presented here can facilitate further modeling, e.g., of electronic structure in nitride alloys. It can be also be helpful in the interpretation of experimental findings.

\section{ACKNOWLEDGMENTS}

This research was supported by the European Union within the European Regional Development Fund through the grant Innovative Economy POIG.01.01.02-00-008/08. Pictures of atomistic structures were generated using the ATOMEYE program. 55
1 N. A. El-Masry, E. L. Piner, S. X. Liu, and S. M. Bedair, Appl. Phys. Lett. 72, 40 (1998)

2 M. D. McCluskey, L. T. Romano, B. S. Krusor, D. P. Bour, N. M. Johnson, and S. Brennan, Appl. Phys. Lett. 72, 1730 (1998)

3 Z. Liliental-Weber, D. Zakharov, K. Yu, J. A. III, W. Walukiewicz, E. Haller, H. Lu, and W. Schaff, Physica B 376-377, 468 (2006), proceedings of the 23rd International Conference on Defects in Semiconductors
${ }^{4}$ K. Pakuła, J. Borysiuk, R. Bożek, and J. Baranowski, J. Cryst. Growth 296, 191 (2006)

${ }^{5}$ M. J. Galtrey, R. A. Oliver, M. J. Kappers, C. J. Humphreys, D. J. Stokes, P. H. Clifton, and A. Cerezo, Appl. Phys. Lett. 90, 061903 (2007)

${ }^{6}$ V. B. Özdöl, C. T. Koch, and P. A. van Aken, J. Appl. Phys. 108, 056103 (2010)

C. J. Humphreys, Philos. Mag. 87, 1971 (2007) 
8 T. M. Smeeton, M. J. Kappers, J. S. Barnard, M. E. Vickers, and C. J. Humphreys, Appl. Phys. Lett. 83, 5419 (2003)

9 C. Kisielowski and T. P. Bartel, Appl. Phys. Lett. 91, 176101 (2007)

10 T. P. Bartel, P. Specht, J. C. Ho, and C. Kisielowski, Philos. Mag. 87, 1983 (2007)

${ }^{i 1}$ L. Bellaiche and A. Zunger, Phys. Rev. B 57, 4425 (1998)

12 S. V. Dudiy and A. Zunger, Phys. Rev. B 68, 041302 (2003)

13 I. Gorczyca, T. Suski, N. E. Christensen, and A. Svane, Phys. Status Solidi C 6 (2009), doi: "bibinfo doi $10.1002 /$ pssc. 200880890

${ }^{14}$ I. Gorczyca, S. P. Łepkowski, T. Suski, N. E. Christensen, and A. Svane, Phys. Rev. B 80, 075202 (2009)

15 I. Gorczyca, T. Suski, N. E. Christensen, and A. Svane, Appl. Phys. Lett. 96, 101907 (2010)

16 S. Chichibu, T. Azuhata, T. Sota, and S. Nakamura, Appl. Phys. Lett. 70, 2822 (1997)

${ }^{1}$ S. F. Chichibu, A. Uedono, T. Onuma, B. A. Haskell, A. Chakraborty, T. Koyama, P. T. Fini, S. Keller, S. P. DenBaars, J. S. Speck, U. K. Mishra, S. Nakamura, S. Yamaguchi, S. Kamiyama, H. Amano, I. Akasaki, J. Han, and T. Sota, Nat. Mater. 5, 810 (2006)

18 M. G. Ganchenkova, V. A. Borodin, K. Laaksonen, and R. M. Nieminen, Phys. Rev. B 77, 075207 (2008)

19 I. Ho and G. B. Stringfellow, Appl. Phys. Lett. 69, 2701 (1996)

20 J. Adhikari and D. A. Kofke, J. Appl. Phys. 95, 4500 (2004)

21 K. Biswas, A. Franceschetti, and S. Lany, Phys. Rev. B 78, $085212(2008)$

${ }^{22}$ L. K. Teles, J. Furthmüller, L. M. R. Scolfaro, J. R. Leite, and F. Bechstedt, Phys. Rev. B 62, 2475 (2000)

${ }^{23}$ L. K. Teles, L. M. R. Scolfaro, J. R. Leite, J. Furthmüller, and F. Bechstedt, J. Appl. Phys. 92, 7109 (2002)

24 C. Caetano, L. K. Teles, M. Marques, A. DalPino, and L. G. Ferreira, Phys. Rev. B 74, 045215 (2006)

25 J. A. Purton, M. Y. Lavrentiev, and N. L. Allan, J. Mater. Chem. 15, 785 (2005)

20 M. Ferhat and F. Bechstedt, Phys. Rev. B 65, 075213 (2002)

27 T. Takayama, M. Yuri, K. Itoh, T. Baba, and J. Harris, Jpn. J. Appl. Phys 39, 5057 (2000)

28 S. Y. Karpov, N. I. Podolskaya, I. A. Zhmakin, and A. I. Zhmakin, Phys. Rev. B 70, 235203 (2004)

29 J. Adhikari and D. A. Kofke, J. Appl. Phys. 95, 6129 (2004)

30 T. Takayama, M. Yuri, K. Itoh, and J. J. S. Harris, J. Appl. Phys. 90, 2358 (2001)

${ }^{31}$ J. Z. Liu and A. Zunger, Phys. Rev. B 77, 205201 (2008)
32 J. Z. Liu and A. Zunger, J. Phys.: Condens. Matter 21, $295402(2009)$

33 J. A. Chan, J. Z. Liu, and A. Zunger, Phys. Rev. B 82, $045112(2010)$

34 S. Y. Karpov, MRS Internet J. Nitride Semicond. Res. 3, $16(1998)$

35 J. M. Ziman, Models of Disorder: The Theoretical Physics of Homogeneously Disordered Systems, 1st ed. (Cambridge University Press, 1979)

${ }^{36}$ F. Ducastelle, Order and Phase Stability in Alloys (Cohesion and Structure) (North Holland, 1991)

37 M. J. Klein, Am. J. Phys 19, 153 (1951)

38 J. M. Cowley, Phys. Rev. 77, 669 (1950)

39 M. P. Allen and D. J. Tildesley, Computer Simulation of Liquids (Oxford University Press, USA, 1989)

40 D. Frenkel and B. Smit, Understanding Molecular Simulatios, 2nd ed. (Academic Press, 2001)

41 P. N. Keating, Phys. Rev. 145, 637 (1966)

42 M. Łopuszyński and J. A. Majewski, J. Phys.: Condens. Matter 22, 205801 (2010)

43 http://www.gnu.org/s/gsl/

44 http://developer.amd.com/libraries/acml

45 S. Chen, X. G. Gong, and S.-H. Wei, Phys. Rev. B 77, 073305 (2008)

46 Butté, J.-F. Carlin, E. Feltin, M. Gonschorek, S. Nicolay, G. Christmann, D. Simeonov, A. Castiglia, J. Dorsaz, H. J. Buehlmann, S. Christopoulos, G. B. H. von Högersthal, A. J. D. Grundy, M. Mosca, C. Pinquier, M. A. Py, F. Demangeot, J. Frandon, P. G. Lagoudakis, J. J. Baumberg, and N. Grandjean, J. Phys. D: Appl. Phys. 40, 6328 (2007)

47 J. Matthews and A. Blakeslee, J. Cryst. Growth 27, 118 (1974)

48 M. E. Sherwin and T. J. Drummond, J. Appl. Phys. 69, 8423 (1991)

49 S. Novikov, N. Stanton, R. Campion, C. Foxon, and A. Kent, J. Cryst. Growth 310, 3964 (2008)

50 S. V. Novikov, C. T. Foxon, and A. J. Kent, Phys. Status Solidi A 207, 1277 (2010)

${ }^{51}$ S. V. Novikov, N. Zainal, A. V. Akimov, C. R. Staddon, A. J. Kent, and C. T. Foxon, J. Vac. Sci. Technol. B 28, C3B1 (2010)

52 S.-H. Wei, L. G. Ferreira, and A. Zunger, Phys. Rev. B 41, $8240(1990)$

53 J. Z. Liu, G. Trimarchi, and A. Zunger, Phys. Rev. Lett. 99, 145501 (2007)

54 J. Z. Liu, G. Trimarchi, and A. Zunger, Appl. Phys. Lett. 95, 081901 (2009)

55 J. Li, Modell. Simul. Mater. Sci. Eng. 11, 173 (2003) 